\title{
Mass of different snow crystal shapes derived from fall speed measurements
}

\author{
Sandra Vázquez-Martín ${ }^{1}$, Thomas Kuhn ${ }^{1}$, and Salomon Eliasson ${ }^{2}$ \\ ${ }^{1}$ Luleå University of Technology (LTU). Department of Computer Science, Electrical and Space Engineering. Division of \\ Space Technology, 98 128, Kiruna, Sweden. \\ ${ }_{2}^{2}$ Swedish Meteorological and Hydrological Institute (SMHI), 601 76, Norrköping, Sweden.
}

Correspondence: Thomas Kuhn (thomas.kuhn@ltu.se)

\begin{abstract}
.
Meteorological forecast and climate models require good knowledge of the microphysical properties of hydrometeors and the atmospheric snow and ice crystals in clouds. For instance, their size, cross-sectional area, shape, mass, and fall speed. Especially shape is an important parameter in that it strongly affects the scattering properties of ice particles, and consequently their response to remote sensing techniques. The fall speed and mass of ice particles are other important parameters both for numerical forecast models and for the representation of snow and ice clouds in climate models. In the case of fall speed, it is responsible for the rate of removal of ice from these models. The particle mass is a key quantity that connects the cloud microphysical properties to radiative properties. Using an empirical relationship between the dimensionless Reynolds and Best numbers, fall speed and mass can be derived from each other if particle size and cross-sectional area are also known.

In this work, ground-based in-situ measurements of snow particle microphysical properties are used to analyse mass as a function of shape and the other properties particle size, cross-sectional area, and fall speed. The measurements for this study were done in Kiruna, Sweden during snowfall seasons of 2014 to 2019 and using the ground-based in-situ instrument Dual Ice Crystal Imager (D-ICI), which takes high-resolution side- and top-view images of natural hydrometeors. From these images, particle size (maximum dimension), cross-sectional area, and fall speed of individual particles are determined. The particles are shape classified according to the scheme presented in our previous work, in which particles sort into 15 different shape groups depending on their shape and morphology. Particle masses of individual ice particles are estimated from measured particle size, cross-sectional area, and fall speed. The selected dataset covers sizes from about $0.1 \mathrm{~mm}$ to $3.2 \mathrm{~mm}$, fall speeds from $0.1 \mathrm{~m} \mathrm{~s}^{-1}$ to $1.6 \mathrm{~m} \mathrm{~s}^{-1}$, and masses from $0.2 \mu \mathrm{g}$ to $320 \mu \mathrm{g}$. In our previous work, the fall speed relationships between particle size and cross-sectional area were studied. In this work, the same dataset is used to determine the particle mass, and consequently, the mass relationships between particle size, cross-sectional area, and fall speed are studied for these 15 shape groups. Furthermore, the mass relationships presented in this study are compared with the previous studies.
\end{abstract}

Keywords: Natural snow crystals; hydrometeors; microphysical properties; fall speed; mass; ground-based in-situ measurements. 


\section{Introduction}

Atmospheric models need accurate knowledge of atmospheric ice crystals and snow particles' microphysical properties to ensure realistic parameterizations (e.g., Stoelinga et al., 2003; Tao et al., 2003). These properties, including size, cross-sectional area, shape, fall speed, and mass of ice particles, cannot be measured directly with remote sensing methods. Therefore, retrieval methods of cloud and snow properties also rely on good assumptions of the microphysical properties.

Particle shape is an essential parameter for retrievals of cloud properties from optical remote sensing (see, e.g., Yang et al., 2008; Baum et al., 2011; Xie et al., 2011; Loeb et al., 2018). Furthermore, it can affect active and passive microwave measurements of clouds and snowfall (e.g., Sun et al., 2011; Matrosov et al., 2012; Marchand et al., 2013; Kneifel et al., 2010; Cooper et al., 2017). Therefore, the shape dependence of the other microphysical properties is crucial to ensure accurate parameterizations. The fall speed of ice and snow crystals is a critical parameter for the modelling of the microphysical precipitation processes (Schefold et al., 2002) and the climate as it influences the lifetime of cirrus clouds, the vertical transport of water, and the snowfall rate (e.g., Mitchell et al., 2008). Ice particle mass parameterizations are required to derive ice water content (IWC). IWC is a crucial parameter to describe cloud contribution to the atmospheric models' radiation budget (Waliser et al., 2009; Thornberry et al., 2017).

Therefore, it is desirable to have datasets of falling snow particles based on simultaneous measurements of the microphysical properties maximum dimension (particle size), cross-sectional area, shape, fall speed, and particle mass. If not available as measurement, particle mass or fall speed is retrievable based on all other properties. The fall speed cannot be computed directly from maximum dimension, cross-sectional area, and mass, because the drag force on the particle depends on the drag coefficient $C_{\mathrm{D}}$ that also depends on the fall speed. The dimensionless Best number $X$ that only depends on maximum dimension, cross-sectional area, and mass can eliminate this interdependency. The Best number can then help determine the Reynolds number, Re, through empirical relationships between Re and $X$. Finally, Re is used to calculate the fall speed, $v$.

For spherical particles, this Re- $X$ relationship is well known (Abraham, 1970). Böhm (1989) suggested a modified Re- $X$ relationship to determine $v$ for all snow particles. Mitchell (1996) used that relationship to derive $v$ vs maximum dimension power laws from dimensional power laws of cross-sectional area and mass. The same $\operatorname{Re}-X$ relationship may also be used to determine mass if $v$ in addition to maximum dimension and cross-sectional area is known. Szyrmer and Zawadzki (2010) have done this to determine average $v$ vs mass relationships from measurements of snow aggregates' fall speeds.

In this study, the masses of individual particles are derived from measured maximum dimensions, cross-sectional areas, and fall speeds using the dataset from our previous study Vázquez-Martín et al. (2020b) that also includes particle shape. We analyse mass relationships as functions of maximum dimension, cross-sectional area, and fall speed for different snow particle shapes. Section 2 describes the dataset used in this work. Section 3 shows the derivation of particle mass and mass relationships. Section 4 shows and discusses the resulting relationship between mass, size, cross-sectional area, and fall speed. 
All relationships are studied separately for various particle shapes. In the same section, we also present comparisons between our mass relationships and those from previous studies. In Sect. 5, this study is summarized and concluded.

\section{Dataset}

The dataset consists of 2461 high-resolution dual images of falling natural snow crystals and other hydrometeors. The data have been collected using D-ICI, the ground-based in-situ instrument described in Kuhn and Vázquez-Martín (2020), at a site in Kiruna, Sweden $\left(67.83^{\circ} \mathrm{N}, 20.41^{\circ} \mathrm{E}\right)$, described in Vázquez-Martín et al. (2020a) during multiple snowfall seasons, the winters of 2014/2015 to 2018/2019. Snow particles are imaged simultaneously from two different viewing directions. One is horizontal, recording a side view, and one is close to vertical, recording a top view. From the top-view images, we can determine for each particle its maximum dimension $D_{\max }$, which we use to describe particle size, cross-sectional area $A$, and area ratio. From the side-view images, since they are exposed twice, we can determine fall speed. These images are highresolution (optical resolution of about $10 \mu \mathrm{m}$ ) where one pixel corresponds to $3.7 \mu \mathrm{m}$. The additional information dual images provide, improves the shape classification carried out by looking at both top- and side-view images. The particles are classified according to their shape and sorted into 15 different shape groups as described in Vázquez-Martín et al. (2020a). A complete description of the dataset and data processing methods is given by Vázquez-Martín et al. (2020b).

\section{Methods}

\subsection{Mass derivation}

The motion of hydrometeors when free-falling through the atmosphere establishes an equilibrium between two forces; the gravity and the aerodynamic drag. The resulting particle settling speed is called fall speed $v$. Thus, the fall speed is governed by the physical properties of the hydrometeors, including their mass and projected area, and in addition it involves aerodynamic principles and environmental conditions. The gravitational force is proportional to the particle mass $m$, while the frictional or drag force is proportional to both the particle projected area, i.e. the cross-sectional area $A$, and the square of its fall speed $v$. The force balance yields

$m \cdot g=\frac{1}{2} \cdot \rho_{\mathrm{a}} \cdot v^{2} \cdot A \cdot C_{\mathrm{D}}$,

where $g$ is the gravitational acceleration, $\rho_{\mathrm{a}}$ the air density, and $C_{\mathrm{D}}$ the drag coefficient. To determine $v$ from the particle properties $m$ and $A$ using this equation, the drag coefficient $C_{\mathrm{D}}$ has to be known as well. However, $C_{\mathrm{D}}$ depends on maximum dimension, shape, and on $v$ itself. To circumvent these interdependencies, one can first determine the Best number $X=C_{\mathrm{D}} \cdot \operatorname{Re}^{2}$ by rearranging Eq. 1 together with the Reynolds number

$\operatorname{Re}=\frac{\rho_{\mathrm{a}} \cdot v \cdot D_{\max }}{\eta}$ 
where $\eta$ is the dynamic viscosity of air, we get the expression that does not depend on fall speed $v$ :

$85 X=\frac{2 \cdot m \cdot g \cdot \rho_{\mathrm{a}} \cdot D_{\max }^{2}}{A \cdot \eta^{2}}$.

Thus, $X$ can be calculated from the particle properties $D_{\max }, A$, and $m$. If the relationship between $\operatorname{Re}$ and $X$ is known, one can determine Re from $X$. In these circumstances, Eq. 2 provides the fallspeed, $v$. Böhm (1989) provides a relationship between Re and $X$ for snow particles, which is shown here in the form given by Mitchell (1996)

$\operatorname{Re}=\frac{\delta_{0}^{2}}{4} \cdot\left[\left(1+\frac{4 \cdot X^{1 / 2}}{\delta_{0}^{2} \cdot C_{0}^{1 / 2}}\right)^{1 / 2}-1\right]^{2}$,

where $\delta_{0}=5.83$ and $C_{0}=0.6$ are unit-less constants, and uses it together with the approach described above to determine $v$ from the particle properties $D_{\max }, A$, and $m$.

In a similar approach, one can determine particle mass if $D_{\max }, A$, and $v$ are known. For this, Re is determined from $v$ and $D_{\max }$ using Eq. 2. Then, $X$ is determined from Eq. 4 solved for $X$

$X=\frac{\delta_{0}^{4} \cdot C_{0}}{16} \cdot\left\{\left[\left(\frac{4 \cdot \operatorname{Re}}{\delta_{0}^{2}}\right)^{1 / 2}+1\right]^{2}-1\right\}^{2}$.

$m=\frac{X \cdot A \cdot \eta^{2}}{2 \cdot g \cdot \rho_{\mathrm{a}} \cdot D_{\max }^{2}}$,

where the atmospheric conditions can be accounted for each particle by adapting $\eta$ and $\rho_{\mathrm{a}}$ to the measured temperature and pressure.

For all non-spherical shapes, i.e. our shape groups (1) Needles, (2) Crossed-needles, (3) Thick columns, (4) Capped columns, (5) Plates, (6) Stellar, (7) Bullet rosettes, (8) Branches, (9) Side planes, (10) Spatial plates, (11) Spatial stellar, (12) Graupel, (13) Ice particles, and (14) Irregulars, the set of $C_{0}=0.6$ and $\delta_{0}=5.83$ that is adapted to fit best all types of snow particles (Böhm, 1989; Mitchell, 1996) is used in Eq. 5. For spherical shapes it is more appropriate to use the constants first introduced by Abraham (1970) for spheres: $C_{0}=0.292$ and $\delta_{0}=9.06$. Thus, for spherical particles in our dataset, i.e. for shape group (15) Spherical, these constants are used instead.

\subsection{Fitting relationships to data}

Once mass is calculated, we can parameterize the relationships mass vs maximum dimension, $m\left(D_{\max }\right)$, mass vs crosssectional area, $m(A)$, and fall speed vs mass, $v(m)$, by fitting our data to the power laws

$m\left(D_{\max }\right)=\tilde{a}_{D} \cdot\left(\frac{D_{\max }}{1 \mathrm{~mm}}\right)^{\tilde{b}_{D}}$

$110 m(A)=\tilde{a}_{A} \cdot\left(\frac{A}{1 \mathrm{~mm}^{2}}\right)^{\tilde{b}_{A}}$, 
$v(m)=a_{m} \cdot\left(\frac{m}{1 \mu \mathrm{g}}\right)^{b_{m}}$

which represent straight lines on logarithmic plots. Hence, linear least-squares fits to the logarithm of the data yield the parameters $\tilde{a}_{D}, \tilde{b}_{D}, \tilde{a}_{A}, \tilde{b}_{A}, a_{m}$, and $b_{m}$. The parameter $\tilde{a}_{D}$ corresponds to the mass at $D_{\max }=1 \mathrm{~mm}, \tilde{a}_{A}$ to the mass at $A=1 \mathrm{~mm}{ }^{2}$, and $a_{m}$ to the fall speed at $m=1 \mu \mathrm{g}$. The parameters $\tilde{b}_{D}, \tilde{b}_{A}$, and $b_{m}$ are the exponents in the power laws and the slopes in the linear fits.

The relationships are determined by binning the data first, before fitting to Eq. 7-Eq. 9. As seen in Vázquez-Martín et al. (2020b), using binned data instead of individual data reduces the data spread so that fit-functions based on binned data are more robust than fit-functions based on individual data. Therefore, also here the data are first binned into a suitable number of bins. Ten mass bins (for $m$ vs $D_{\max }$ and $m$ vs $A$ relationships) and ten fall speed bins (for $v$ vs $m$ ) are used, respectively. The bins are spaced such that each bin contains as close to the same number of particles as possible. As a consequence, the bin widths are variable and specific to each shape group, and thereby avoid the problem of individual bins having a disproportional effect on the fit. The binned data consist of the median values for each bin. Then, the $m$ vs $D_{\max }, m$ vs $A$, and $v$ vs $m$ relationships are fitted to the median masses vs median maximum dimensions, median masses vs median cross-sectional areas, and median fall speeds vs median masses, respectively.

\subsection{Analytical derivation of relationships}

These relationships may be useful for parameterizations in models and retrievals and are readily comparable to other studies. In case a suitable dataset is not available, an alternative to fitting these relationships to measured data, is to derive particle mass analytically from previously determined parameterizations of cross-sectional area vs maximum dimension ( $A$ vs $\left.D_{\text {max }}\right)$, fall speed vs maximum dimension ( $v$ vs $\left.D_{\max }\right)$ and fall speed vs cross-sectional area $(v$ vs $A)$ given by power laws

$$
\begin{aligned}
& A\left(D_{\max }\right)=a \cdot\left(\frac{D_{\max }}{1 \mathrm{~mm}}\right)^{b}, \quad D_{\max }(A)=a^{\prime} \cdot\left(\frac{A}{1 \mathrm{~mm}^{2}}\right)^{b^{\prime}}, \\
& v\left(D_{\max }\right)=a_{D} \cdot\left(\frac{D_{\max }}{1 \mathrm{~mm}}\right)^{b_{D}}, \quad D_{\max }(v)=a_{D}^{\prime} \cdot\left(\frac{v}{1 \mathrm{~m} \mathrm{~s}^{-1}}\right)^{b_{D}^{\prime}},
\end{aligned}
$$

$v(A)=a_{A} \cdot\left(\frac{A}{1 \mathrm{~mm}^{2}}\right)^{b_{A}}, \quad A(v)=a_{A}^{\prime} \cdot\left(\frac{v}{1 \mathrm{~m} \mathrm{~s}^{-1}}\right)^{b_{A}^{\prime}}$.

For each relationship, the inverse is also shown as the corresponding parameters are convenient for some of the derivations. The parameter $a$ corresponds to the cross-sectional area at $D_{\max }=1 \mathrm{~mm}, a^{\prime}$ corresponds to the maximum dimension at $A=$ $1 \mathrm{~mm}^{2}, a_{D}$ to the fall speed at $D_{\max }=1 \mathrm{~mm}, a_{D}^{\prime}$ to the maximum dimension at $v=1 \mathrm{~m} \mathrm{~s}^{-1}, a_{A}$ to the fall speed at $A=$ $1 \mathrm{~mm}^{2}$, and $a_{A}^{\prime}$ to the cross-sectional area at $v=1 \mathrm{~m} \mathrm{~s}^{-1}$. The parameters $b, b^{\prime}, b_{D}, b_{D}^{\prime}, b_{A}$, and $b_{A}^{\prime}$ are the exponents in the power laws. 
The resulting power laws are

$$
\begin{aligned}
& m\left(D_{\max }\right)=\frac{\eta^{2} \cdot \gamma \cdot a}{2 \cdot g \cdot \rho_{\mathrm{a}} \cdot 1 \mathrm{~mm}^{2}} \cdot\left(\frac{a_{D} \cdot \rho_{\mathrm{a}} \cdot 1 \mathrm{~mm}}{\eta}\right)^{\delta} \cdot\left(\frac{D_{\max }}{1 \mathrm{~mm}}\right)^{b_{D} \cdot \delta+\delta+b-2}, \\
& m(A)=\frac{\gamma \cdot 1 \mathrm{~mm}^{2} \cdot \eta^{2}}{2 \cdot g \cdot a^{\prime 2} \cdot \rho_{\mathrm{a}}} \cdot\left(\frac{a_{A} \cdot a^{\prime} \cdot \rho_{\mathrm{a}}}{\eta}\right)^{\delta} \cdot\left(\frac{A}{1 \mathrm{~mm}^{2}}\right)^{b_{A} \cdot \delta+b^{\prime} \cdot \delta+1-2 \cdot b^{\prime}},
\end{aligned}
$$

$v(m)=\left[\frac{1 \mu \mathrm{g} \cdot 2 \cdot g \cdot a_{D}^{\prime 2} \cdot \rho_{\mathrm{a}}}{\gamma \cdot a_{A}^{\prime} \cdot \eta^{2}} \cdot\left(\frac{\eta}{a_{D}^{\prime} \cdot \rho_{\mathrm{a}} \cdot 1 \mathrm{~m} \mathrm{~s}^{-1}}\right)^{\delta}\right]^{\frac{\delta}{\left(b_{D}^{\prime}+1\right) \cdot \delta+b_{A}^{\prime}-2 \cdot b_{D}^{\prime}}} \cdot\left(\frac{m}{1 \mu \mathrm{g}}\right)^{\frac{1}{\left(b_{D}^{\prime}+1\right) \cdot \delta+b_{A}^{\prime}-2 \cdot b_{D}^{\prime}}}$

The derivation of these power laws is shown in Appendix B (Eq. B3-Eq. B6). There, also the $X$ vs Re relationship is expressed as power law instead of using Eq. 5. This can be done by approximating Eq. 5 piece-wise in several regions of $X$ to power laws Eq. B1, as done by (Mitchell, 1996). Note, that both methods for deriving the relationships given by Eq. 7-Eq. 9, described in Sect. 3.1 and in this section, are equivalent if they are based on the same dataset. The two methods will yield the same relationships if both use the same power law approximations of $X$ vs $\operatorname{Re}$ and the same atmospheric conditions (given as constant $\eta$ and $\rho_{\mathrm{a}}$ for the whole dataset).

\section{Results and discussions}

\subsection{Results from fitting and correlations}

The particle masses have been determined from measured $D_{\max }, A$, and $v$ with the method described in Sect. 3.1. The $m$ vs $D_{\max }, m$ vs $A$, and $v$ vs $m$ relationships given by Eq. 7-Eq. 9 are then fitted to the resulting data, now consisting of $D_{\max }, A$, $v$, and $m$, for the 15 shape groups using the fitting method based on binned data described in Sect. 3.2. Figure 1 and Table 1 show the results. For simplicity, we use short names included in Table 1 for the shape groups from here on, and Fig. 1 shows their full names. The large spread in the data represented as individual points is apparent in Figs. A1-A3 in Appendix A.

When fitting $m$ vs $D_{\max }, m$ vs $A$, and $v$ vs $m$ relationships to the binned data, we note that, in general, there is a high correlation $\left(0.6 \lesssim R^{2} \lesssim 1\right)$ for most shape groups. In the following, we call the correlation coefficients $R_{D}^{2}, R_{A}^{2}$, and $R_{m}^{2}$ to indicate to which of the three relationships they belong to. For the $m$ vs $D_{\max }$ relationship, the only exceptions to high correlations are shape groups (1) Needles and (2) Crossed needles with $R_{D}^{2} \simeq 0.2$ as well as (3) Thick columns with $R_{D}^{2}=$ 0.51 . For the $m$ vs $A$ relationship, only shape group (10) Spatial plates has a lower correlation with $R_{A}^{2}=0.45$. In these few cases, judging by these low $R^{2}$ values, it is uncertain if the fit functions are representative of the measured data. Figure 2 compares the coefficients $R_{D}^{2}$ and $R_{A}^{2}$ of all the shape groups, and for most shape groups, the two are similar. Only the four groups (1), (2), (3), and (10), mentioned above with lower correlation in one of the relationships, have a distinct difference between $R_{D}^{2}$ and $R_{A}^{2}$. These are clearly above and below the line representing $R_{A}^{2}=R_{D}^{2}$ in Fig. 2 . Of these, the three shape groups (1)-(3) above the line have a better correlation for $m$ vs $A$ than for $m$ vs $D_{\max }$, which is consistent with a better $v$ vs $A$ correlation than $v$ vs $D_{\max }$ for the same groups (Vázquez-Martín et al., 2020b), given that we have derived $m$ using measured $v$ here. 
For the $v$ vs $m$ relationship, all values of $R_{m}^{2}$ are 0.81 or higher. These high values indicate that $v$ is better correlated to $m$ than to $D_{\max }$ or $A$ (see the generally lower $R^{2}$ values reported in Vázquez-Martín et al., 2020b). The generally very high correlations are partly also a consequence of $m$ being derived from $v$, rather than being an independent measurement.

\subsection{Mass versus $D_{\max }$ and $A$}

Figure 1a and Fig. 1b show the $m$ vs $D_{\max }$ and $m$ vs $A$ relationships including, for reference, the mass of liquid water spheres symbolizing rain or fog droplets given by the power laws $m=\frac{\pi}{6} \cdot \rho_{\mathrm{w}} \cdot D_{\max }^{3}$ and $m=\frac{4 \cdot \rho_{\mathrm{w}}}{3 \cdot \sqrt{\pi}} \cdot A^{3 / 2}$, respectively, where $\rho_{\mathrm{w}}=$ $1 \mathrm{~g} \mathrm{~cm}^{-3}$ is the density of liquid water. The mass of spheres is proportional to $D_{\max }^{3}$ and to $A^{3 / 2}$. Thus, comparing to Eq. 7 and Eq. 8, one can see that the exponents $\tilde{b}_{D}=3$ and $\tilde{b}_{A}=1.5$ for spheres. The values of $\tilde{a}_{D}$ and $\tilde{a}_{A}$ for spheres are $524 \mu \mathrm{g}$, the mass of a droplet with $1 \mathrm{~mm}$ diameter, and $752 \mu \mathrm{g}$, the mass of a droplet with a cross-sectional area $A=1 \mathrm{~mm}^{2}$, respectively.

\subsubsection{Slopes $\tilde{b}_{D}$ and $\tilde{b}_{A}$}

The exponent $\tilde{b}_{D}$ for shape groups (12) Graupel and (15) Spherical is close to the value of 3 for spheres, 2.87 and 2.81, respectively. For the same groups, $\tilde{b}_{A}$ is close to the value of 1.5 for spheres, 1.42 for both shape groups. For these shape groups, this is expected due to their spherical or roundish morphology. These exponent values, corresponding to the slopes in Fig. 1a) and b) are among the highest values for all shape groups. Shape groups (6) Stellar and (11) Spatial stellar are the only other shape groups that have similarly steep relationships. These two groups do not have a roundish morphology that could explain this. However, a slope similar to spherical particles indicates that in these groups the morphology remains similar independent of size, i.e. during growth the ice particles grow equally in all three dimensions.

For most other shape groups, the exponent $\tilde{b}_{D}$ varies between 1.2 and 2, and all other $\tilde{b}_{A}$ values range between 0.8 and 1.2. Three shape groups, (1) Needles, (2) Crossed Needles, and (3) Thick columns, stand out with the lowest exponents $\tilde{b}_{D}$ of approximately 0.8 or lower. These can easily be seen in Fig. 1a) as the lines with the most shallow slopes. For these groups, this is understandable due to their morphology. We have seen in Vázquez-Martín et al. (2020b) that an increase in $D_{\max }$ (needle length) is directly proportional to $A$, indicating that the diameter of these needle-shaped particles (needle width) remains similar, whereas $D_{\max }$, and consequently $A$ is growing. Thus, these shapes are clear examples of a size-dependent morphology, i.e. as size increases, not all three dimensions grow at the same rate. In this case, since $D_{\max }$ is approximately proportional to $A$, one would expect both values of $\tilde{b}_{D}$ and $\tilde{b}_{A} \simeq 1$, which most of them are for these three shape groups. Only $\tilde{b}_{D}$ for shape groups (1) and (2) are smaller than 1, indicating a decreasing width as the particle length increases. This seems inconsistent, which might be due to the $X$-Re relationship given by Eq. 5 not being accurate for these shapes. However, this may also be related to the very low correlation in these two cases.

The ratio between the exponents $\tilde{b}_{D}$ and $\tilde{b}_{A}$ is equal to the exponent $b$, as can be seen from Eq. 7, Eq. 8, and Eq. 10. Figure 3 shows the ratios $\frac{\tilde{b}_{D}}{\tilde{b}_{A}}$ plotted vs $b$, and most ratios on this plot are close to the line, $\frac{\tilde{b}_{D}}{\tilde{b}_{A}}=b$, and range between 1.6 to 2 . The exceptions which have ratios much below the line are the two shape groups where $R^{2}$ was relatively low, $R_{D}^{2} \simeq 0.2$ for $m$ vs $D_{\max }$ relationship, i.e. groups (1) Needles and (2) Crossed needles. The ratios for shape groups (3) Thick columns, (9) Side planes, and (13) Ice particles are found slightly below the line, with values between 0.9 and 1.5. Of these groups, (3) and (13) 

regions around the fits. For group (9) Side planes, the uncertainty is smaller and can not explain the lower ratio. Instead, the $X-$ Re relationship given by Eq. 5 not describing well this shape group may be responsible again.

\subsubsection{Coefficients $\tilde{a}_{D}$ and $\tilde{a}_{A}$}

All relationships but those of shape group (15) Spherical form a cluster of lines located in a smaller region in both Figs. 1a) and b). The only relationship found outside this cluster is that of shape group (15), which, if extrapolated towards larger sizes or cross-sectional areas, predicts larger masses than any relationship of the other shape groups. The fit coefficients $\tilde{a}_{D}$ and $\tilde{a}_{A}$ reflect this since they predict the mass at the unit reference of $1 \mathrm{~mm}$ for $\tilde{a}_{D}$ and $1 \mathrm{~mm}^{2}$ for $\tilde{a}_{A}$. These values are much larger for spheres, $\tilde{a}_{D}=244 \mu \mathrm{g}$ and $\tilde{a}_{A}=381 \mu \mathrm{g}$, respectively, than for any other shape group. The second-largest values are for shape group (12) Graupel, $\tilde{a}_{D}=56.0 \mu \mathrm{g}$ and $\tilde{a}_{A}=138 \mu \mathrm{g}$, respectively, all other groups have still much smaller values. The smallest values are found for the five groups (1-3), (6), and (8). Of these, (1-3) form the lower edge of the cluster of all $m$ vs $D_{\max }$ relationships except for group (15) (Fig. 1a), which furthermore have the lowest and most distinct slopes mentioned earlier. Similarly, these five groups (1-3), (6), and (8) form the lower edge of the cluster of all $m$ vs $A$ relationships except for group (15) (Fig. 1b).

As can be seen in Figs. 1a) and b), the power laws for (15) are close to the reference lines for liquid droplets, however, predicting somewhat lower masses. These differences may be due to several reasons. While shape group (15) Spherical may contain liquid droplets, it also contains ice particles that have a lower bulk density $\rho_{\text {ice }}$ compared to the bulk density of liquid water $\rho_{\mathrm{w}}$. Also, the small frozen rain droplets that shape group (15) contains, are not perfectly spherical, which leads to overestimating mass if assuming a spherical shape. Furthermore, sizing errors cause an apparent error in fall speeds. Overestimating the size leads effectively to too low $v$, which in turn yields too low derived $m$.

\subsection{Fall speed versus particle mass}

The exponent values $b_{m}$, i.e. the slopes of the $v$ vs $m$ relationships on Fig. 1c), vary less than the slopes of the $m$ vs $D_{\max }$ and $m$ vs $A$ relationships, they range only from 0.34 to 0.50 . The shape groups with the highest slope values include group (15) as well as most of the groups that had the lowest slope values in the $m$ vs $D_{\max }$ and $m$ vs $A$ relationships, $\tilde{b}_{D}$ and $\tilde{b}_{A}$, respectively, i.e. groups (1-3) and (6). Rather than the slopes, different speeds at any given mass distinguish the different shapes. This can be seen with the values of $a_{m}$, representing the fall speed predicted by the relationships at the mass given by the reference unit of $1 \mu \mathrm{g}$. However, $1 \mu \mathrm{g}$ is below usually encountered masses for most shape groups. Therefore, it is more instructive to evaluate predicted fall speeds closer to the median of masses in the dataset. At a mass of for example $3 \mu \mathrm{g}$, the fall speeds vary between $0.17 \mathrm{~m} \mathrm{~s}^{-1}$ and $0.53 \mathrm{~m} \mathrm{~s}^{-1}$ as seen in Fig. 1c). The highest four fall speeds at this mass correspond to shape groups (15), (13), (3), and (12), in order of descending speed. These groups contain the most compact shapes. Contrarily, the group with the lowest speed at $3 \mu \mathrm{g}$, shape group (6), features the most open structures. 


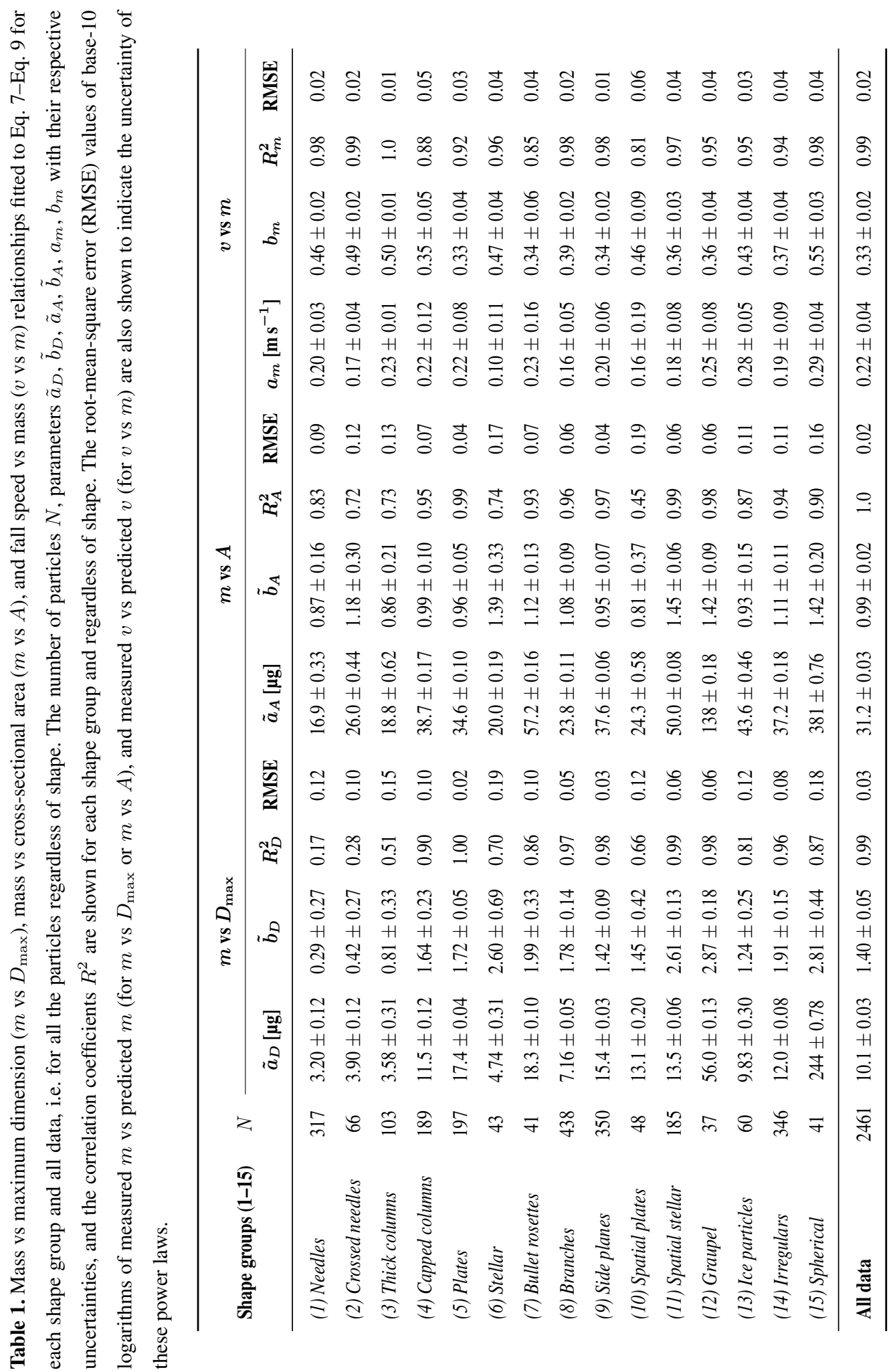



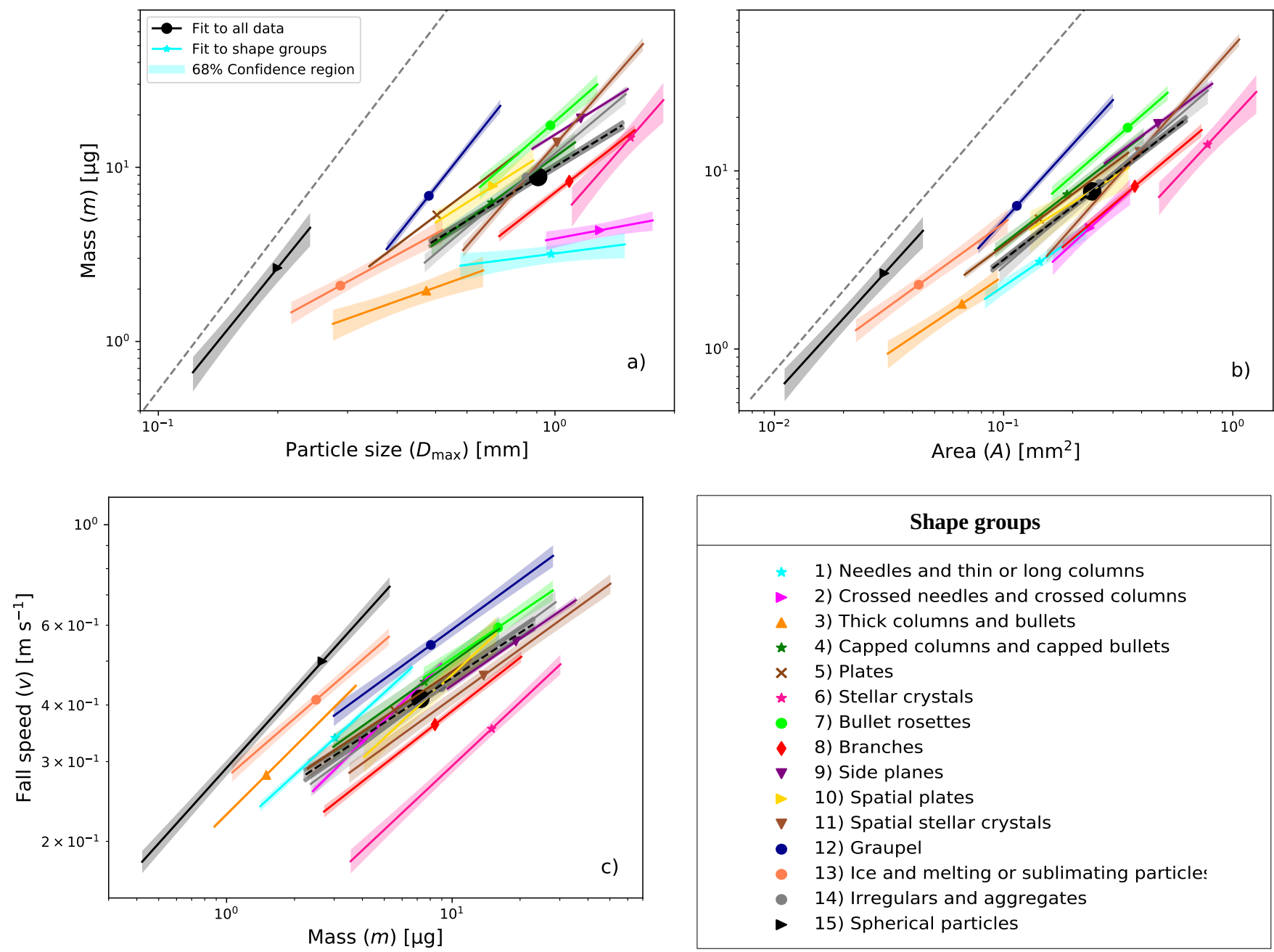

\begin{tabular}{|l}
\multicolumn{1}{c|}{ Shape groups } \\
\hline$\quad$ 1) Needles and thin or long columns \\
$\quad$ 2) Crossed needles and crossed columns \\
$\star \quad$ 3) Thick columns and bullets \\
$\times \quad$ 5) Plates \\
$\star \quad$ 6) Stellar crystals \\
7) Bullet rosettes \\
8) Branches \\
9) Side planes \\
$\nabla$ 10) Spatial plates \\
11) Spatial stellar crystals \\
12) Graupel \\
13) Ice and melting or sublimating particle: \\
14) Irregulars and aggregates
\end{tabular}

Figure 1. Mass vs maximum dimension ( $m$ vs $D_{\max }$ ), mass vs cross-sectional area ( $m$ vs $A$ ), and fall speed vs mass ( $v$ vs $m$ ) relationships are shown in logarithmic scale for all the shape groups (solid lines) and all data (dashed black line). The median $D_{\max }, A$, and $m$ of the data is represented as a single point on each line. The length of fit lines is defined by $16^{\text {th }}$ and $84^{\text {th }}$ percentiles of $D$ max,$A$, and $m$. The $68 \%$ confidence region for the fits is also shown. a) The $m$ vs $D_{\max }$ relationships. For comparison, the mass of spheres, corresponding to rain or fog droplets, given by $m=\frac{\pi}{6} \cdot \rho_{\mathrm{w}} \cdot D_{\text {max }}^{3}$, where the density $\rho_{\mathrm{w}}=1 \mathrm{~g} \mathrm{~cm}^{-3}$, is shown as a grey dashed line. b) The $m$ vs $A$ relationships. For comparison, the mass of spheres given by $m=\frac{4 \cdot \rho_{\mathrm{W}}}{3 \cdot \sqrt{\pi}} \cdot A^{3 / 2}$ is shown as a grey dashed line. c) The $v$ vs $m$ relationships.

\subsection{Comparison with previous studies}

The mass vs particle size ( $m$ vs $D$ ) and fall speed vs mass ( $v$ vs $m$ ) relationships of the common shapes plates, dendrites, graupel, and spheres, i.e. for our shape groups (5) Plates, (6) Stellar (called dendrites in other studies), (12) Graupel, and 


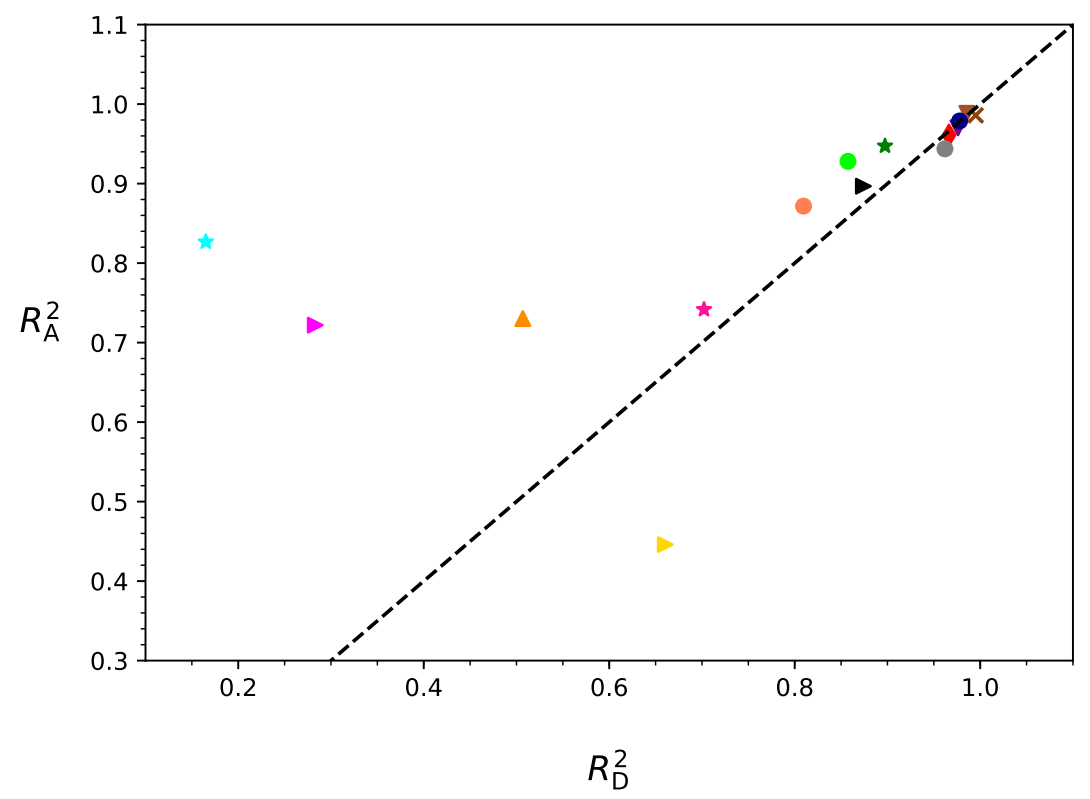

Figure 2. The correlation coefficients $R_{D}^{2}\left(m\right.$ vs $\left.D_{\max }\right)$ and $R_{A}^{2}(m$ vs $A$ ) are shown for the 15 shape groups. For a legend of the shape groups, see Fig. 1. The black dashed line represents $R_{D}^{2}=R_{A}^{2}$.

(15) Spherical, respectively, are compared to previously published relationships based on measurements of mass of individual particles. The parameterizations of $m$ vs $D$ (see Fig. 4a-c) selected for this comparison are taken from Locatelli and Hobbs (1974) [Lo], Heymsfield and Kajikawa (1987) [H], Kajikawa (1989) [K], Mitchell (1996) [M], and Erfani and Mitchell (2017) $[E]$ and are listed in Table 2. For comparison with $v$ vs $m$ (see Fig. 4d) of our shape groups (12) Graupel and (15) Spherical, parameterizations of measurements by [Lo] (see also Table 2) and measurements of Gunn and Kinzer (1949) [G] have been selected. Relationships from this work are further referred to as $[V M]$. Fig. 4 shows all these relationships. For comparison, a line for speeds determined from Eq. 2 using Re calculated from the Re vs $X$ relationship Eq. 4 with $C_{0}=0.292$ and $\delta_{0}=9.06$ and $X$ given by Eq. 3 for spherical particles having a density $\rho_{\mathrm{w}}=1 \mathrm{~g} \mathrm{~cm}^{-3}$ is added to the $v$ vs $m$ relationships in Fig. 4 . This line will be referred to as $[\operatorname{Re}-X]$.

Depending on the study, the particle size $D$ was defined somewhat differently. For $[V M]$, as well as for $[H],[E],[K],[M]$, and $[M a], D$ corresponds to $D_{\max }$. For $[L o], D$ is the diameter of an estimated circle that has the same cross-sectional area as the imaged particle.

\subsubsection{Plates}

We note that for plates (Fig. 4a), the $m$ and $D$ relationship for crystal with sector-like branches (P1b) reported by [M] is most similar to $[V M]$. It is similar also with respect to its slope given by the value of $\tilde{b}_{D}$, all other relationships are steeper with 


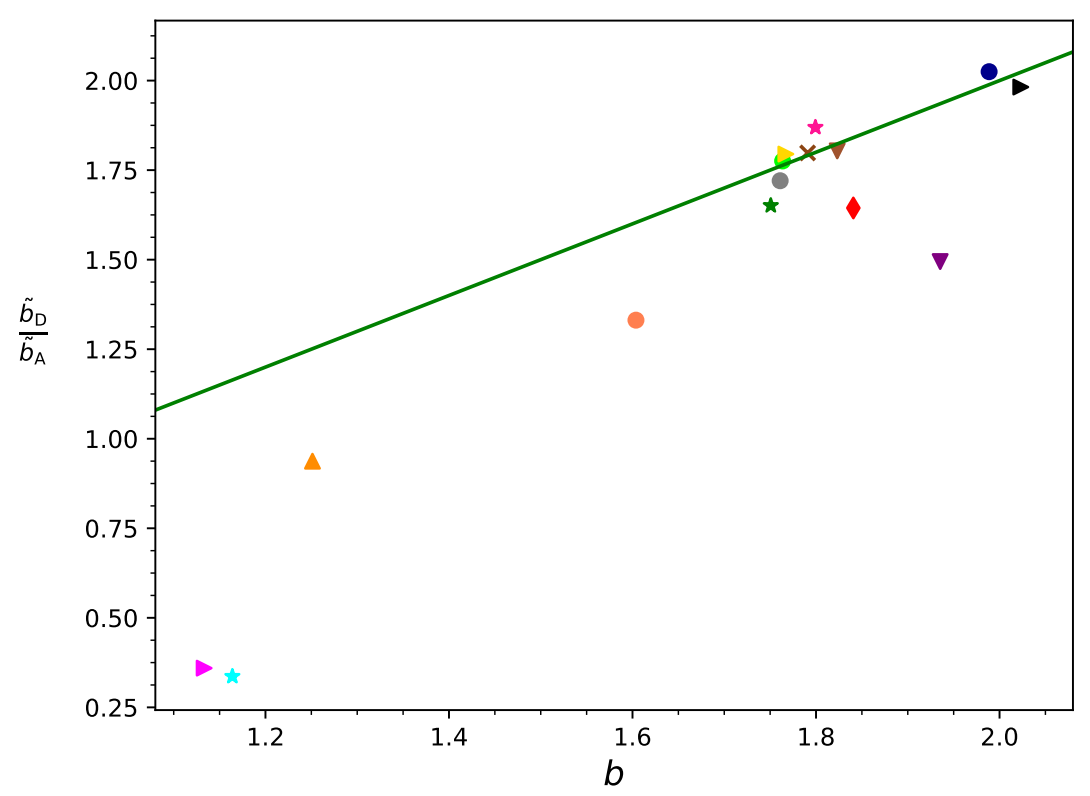

Figure 3. Ratio of the coefficients $\tilde{b}_{\mathrm{D}}$ and $\tilde{b}_{\mathrm{A}}$ from fits to $m$ vs $D_{\max }$ and $m$ vs $A$ relationships, respectively, and the coefficient $b$ corresponding to $A$ vs $D_{\max }$ relationship are shown for all the shape groups. The green solid line represents a reference for spheres, which corresponds to $\frac{\tilde{b}_{\mathrm{D}}}{\tilde{b}_{\mathrm{A}}}=b$.

larger values of $\tilde{b}_{D}$. Reasonably close to $[V M]$ is also the relationship for hexagonal plate by $[M]$, which, however, is heavier at larger sizes than about $1 \mathrm{~mm}$. For those larger sizes hexagonal plate by [M] is similar to hexagonal plate by [H], the latter having the steepest slope (with $\tilde{b}_{D}$ even larger than 3). The relationship for P1b by $[H]$ predicts the lightest particles below about $2 \mathrm{~mm}$. At about $2 \mathrm{~mm}$, it is also similar to [VM] and P1b by [M]. Thick plates by [H] are heavier at larger sizes, but similar to $[V M]$ at about $0.2 \mathrm{~mm}$.

\subsubsection{Dendrites}

For stellar particles (Fig. 4b), several $m$ and $D$ relationships are fairly close to [VM], for example the two relationships for P2a from $[H]$, which has a similar slope, and $[K]$, which has a lower slope than $[V M]$. Relationships for another stellar particle type, P1d, are still relatively close to $[V M]$. For example the one by $[M]$ crosses $[V M]$ somewhat above $1 \mathrm{~mm}$ and is higher for smaller particles, whereas the one by $[H]$ is about a factor of 2 below [VM]. This P1d relationship by $[H]$ may be compared to the rimed stellars (R1d) of the same study [H]. These two curves differ by about a factor of two, with the rimed stellar having the larger mass and being very close to $[V M]$. Riming of stellar particles adds mass without increasing their size noticeably (Erfani and Mitchell, 2017) as seen in [E], which explains the difference seen between the two mentioned relationships of [H]. A similar difference is seen between the two relationships by $[E]$ from a dataset used to study effects of riming. However, the two 
relationships by $[K]$, which also feature unrimed and rimed stellar particles, respectively, do not show a significant difference. Particles included in shape group (6) Stellar of $[V M]$ include cases of light riming. Distinguishing between unrimed and rimed stellar particles in the data of group (6) resulted in two relationships (not shown) that are both, within uncertainties, identical to the one produced from all data in shape group (6).

\subsubsection{Graupel and spheres}

[Lo] reported three $m$ vs $D$ relationships for lump graupel (R4b) corresponding to three different particle densities with larger masses predicted by the relationships for higher densities. Our relationship for graupel is between [Lo]'s low and medium density relationships (Fig. 4c). It is well approximated, by the mass of spherical particles with a density of $0.12 \mathrm{~g} \mathrm{~cm}^{-3}$, which is at the lower end of the density range reported by [Lo] for their medium density relationship $\left(>0.10\right.$ to $\left.0.25 \mathrm{~g} \mathrm{~cm}^{-3}\right)$. The relationship by $[H]$ for lump graupel (R4b) is similar to [Lo]'s medium density. The relationship by [E] agrees also with [VM], but only around $1 \mathrm{~mm}$, as their relationship has a much lower slope $\left(\tilde{b}_{D}=2.16\right)$ than all other relationships for graupel (2.7 to 3.1). The mass of liquid water spheres $m=\frac{\pi}{6} \cdot \rho_{\mathrm{w}} \cdot D^{3}$ that was shown on Fig. 1a) is added also to Fig. 4c) as reference. Its comparison with [VM]'s line for shape group (15) Spherical is discussed in Sect. 4.2.2.

The $v$ vs $m$ relationships from [Lo] (Fig. 4d) come, within their ranges, close to our relationship for shape group (12). In general, at a certain particle mass, the size and cross-sectional area, and thus the drag force, decrease with increasing graupel particle density. This can be seen, to some extent, for the three lines by [Lo]. However, their lines have different slopes in a way that makes them intersect with each other. Their slopes are more shallow than the relationship of $[V M]$, consequently they also cross that line. The slope for graupel of $[V M]$ is more similar to that of the relationships related to spherical particles than the lines for graupel by $[\mathrm{Lo}]$. Consequently it approaches spherical particles, which represent an upper limit in speed, at a lower mass than the lines by $[L o]$.

The lines for spherical particles of $[G]$ and $[\operatorname{Re}-X]$ are very close to each other, thus $[\operatorname{Re}-X]$ predicts well these measurements. The straight line for the shape group (15) of $[V M]$ is at somewhat lower fall speeds below approximately $10 \mu \mathrm{g}$ and at higher speeds above that mass. It represents the power-law fit to our measurements of droplets and spherical and almost spherical ice particles, whereas the two curved lines of $[G]$ and $[\operatorname{Re}-X]$ represent only liquid droplets.

\section{Summary and conclusions}

This manuscript presents new mass and fall speed parameterizations derived from D-ICI measurements of natural snow, ice crystals and other hydrometeors, covering sizes from $0.1 \mathrm{~mm}$ to $3.2 \mathrm{~mm}$. Using the dataset and fall speed vs maximum dimension and vs cross-sectional area relationships from Vázquez-Martín et al. (2020b), where fall speeds cover ranges from $0.1 \mathrm{~m} \mathrm{~s}^{-1}$ to $1.6 \mathrm{~m} \mathrm{~s}^{-1}$, in this work, we have added particle masses to our dataset of measured maximum dimension, crosssectional area, and fall speed of individual particles. The calculated values of individual particle masses range from $0.2 \mu \mathrm{g}$ to $320 \mu \mathrm{g}$. 
Table 2. The $m$ vs $D$ and $v$ vs $m$ relationships of previous studies given by Locatelli and Hobbs (1974) [Lo], Heymsfield and Kajikawa (1987) [H], Kajikawa (1989) [K], Mitchell (1996) [M], and Erfani and Mitchell (2017) [E] are shown for some shapes that were selected for the comparison and correspond to (5) Plates, (6) Stellar, (12) Graupel, and (15) Spherical. The power laws for [M] have been determined by using equation [15] in Mitchell (1996). The relationships found in this work are also shown as [VM]. The snow particles type, the total number of particles $N$, ranges of particle sizes $D$, mass $m$, fall speeds $v$, the $m$ vs $D, v$ vs $m$ relationships, the correlation coefficient ( $R^{2}$ ), and the reference of the studies are displayed. In these references, the particle sizes are defined somewhat differently. In [VM], as well as $[H]$ and $[M], D$ is defined as $D_{\max }$. Magono and Lee (1966)'s symbols are sometimes added for shape clarification. These $m$ vs $D, v$ vs $m$ relationships are shown in Fig. 4. The power laws from the literature have been converted in order to have the same units, so that mass $m$ is in $\mu \mathrm{g}$, particle size $D$ in $\mathrm{mm}$, and fall speed $v$ in $\mathrm{m} \mathrm{s}^{-1}$.

\begin{tabular}{|c|c|c|c|c|c|c|c|}
\hline Snow particle type & $N$ & Range of $D$ & Range of $m$ & Range of $v$ & Relationships $(m-D, v-m)$ & $R^{2}$ & Ref. \\
\hline Shape group (5) Plates & 197 & $0.21-1.7 \mathrm{~mm}$ & $0.53-62 \mu \mathrm{g}$ & $0.11-0.9 \mathrm{~m} \mathrm{~s}^{-1}$ & 1. $m /(\mu \mathrm{g})=17.4 \cdot(D / \mathrm{mm})^{1.72}$ & 1.0 & [VM] \\
\hline Hexagonal plates & - & $0.10-3.0 \mathrm{~mm}$ & - & - & 2. $m /(\mu \mathrm{g})=26.2 \cdot(D / \mathrm{mm})^{2.45}$ & - & {$[M]$} \\
\hline Thick plate $(\mathrm{C} 1 \mathrm{~h})$ & 19 & $0.30-0.6 \mathrm{~mm}$ & $2.6-10 \mu \mathrm{g}$ & - & 4. $m /(\mu \mathrm{g})=54.9 \cdot(D / \mathrm{mm})^{2.68}$ & 0.67 & {$[\mathrm{H}]$} \\
\hline Hexagonal plate (P1a) & 34 & $0.30-1.5 \mathrm{~mm}$ & $0.20-70 \mu \mathrm{g}$ & - & 5. $m /(\mu \mathrm{g})=18.4 \cdot(D / \mathrm{mm})^{3.31}$ & 0.93 & {$[H]$} \\
\hline Stellar crystal with broad arms (P1d) & - & $0.09-1.5 \mathrm{~mm}$ & - & - & 8. $m /(\mu \mathrm{g})=5.77 \cdot(D / \mathrm{mm})^{1.67}$ & - & {$[M]$} \\
\hline Stellar crystal with broad arms (P1d) & 23 & $0.40-2.4 \mathrm{~mm}$ & $0.20-31 \mu \mathrm{g}$ & - & 9. $m /(\mu \mathrm{g})=2.47 \cdot(D / \mathrm{mm})^{2.59}$ & 0.95 & {$[H]$} \\
\hline Stellar with end plates (P2a) & 11 & $0.70-3.0 \mathrm{~mm}$ & 4.9-92 $\mu \mathrm{g}$ & - & 10. $m /(\mu \mathrm{g})=6.23 \cdot(D / \mathrm{mm})^{2.53}$ & 0.88 & {$[H]$} \\
\hline Rimed stellar (R1d) & 48 & $0.70-5.3 \mathrm{~mm}$ & $2.0-539 \mu \mathrm{g}$ & - & 11. $m /(\mu \mathrm{g})=5.34 \cdot(D / \mathrm{mm})^{2.58}$ & 0.85 & {$[H]$} \\
\hline Rimed stellar (R1d) & 43 & $1.6-5.8 \mathrm{~mm}$ & - & - & 15. $m /(\mu \mathrm{g})=9.18 \cdot(D / \mathrm{mm})^{1.76}$ & $r=0.68$ & {$[K]$} \\
\hline \multirow[t]{2}{*}{ Shape group (12) Graupel } & 37 & $0.25-1.2 \mathrm{~mm}$ & $1.10-75 \mu \mathrm{g}$ & $0.26-1.0 \mathrm{~m} \mathrm{~s}^{-1}$ & 16. $m /(\mu \mathrm{g})=56.0 \cdot(D / \mathrm{mm})^{2.87}$ & 0.98 & {$[V M]$} \\
\hline & 37 & $0.25-1.2 \mathrm{~mm}$ & $1.10-75 \mu \mathrm{g}$ & $0.26-1.0 \mathrm{~m} \mathrm{~s}^{-1}$ & 17. $v /\left(\mathrm{m} \mathrm{s}^{-1}\right)=0.25 \cdot(\mathrm{m} / \mu \mathrm{gg})^{0.36}$ & 0.95 & {$[V M]$} \\
\hline \multirow[t]{2}{*}{ Lump graupel (R4b) } & 35 & $0.50-2.0 \mathrm{~mm}$ & - & - & 18. $m /(\mu \mathrm{g})=42.0 \cdot(D / \mathrm{mm})^{3.00}$ & $r=0.98$ & [Lo] \\
\hline & 35 & $0.50-2.0 \mathrm{~mm}$ & - & - & 19. $v /\left(\mathrm{m} \mathrm{s}^{-1}\right)=0.46 \cdot(\mathrm{m} / \mu \mathrm{g})^{0.15}$ & $r=0.53$ & [Lo] \\
\hline \multirow[t]{2}{*}{ Lump graupel (R4b) } & 58 & $0.50-3.0 \mathrm{~mm}$ & - & - & 20. $m /(\mu \mathrm{g})=78.0 \cdot(D / \mathrm{mm})^{2.80}$ & $r=0.93$ & [Lo] \\
\hline & 58 & $0.50-3.0 \mathrm{~mm}$ & - & - & 21. $v /\left(\mathrm{m} \mathrm{s}^{-1}\right)=0.46 \cdot(\mathrm{m} / \mu \mathrm{g})^{0.24}$ & $r=0.84$ & [Lo] \\
\hline \multirow[t]{2}{*}{ Lump graupel (R4b) } & 17 & $0.50-1.0 \mathrm{~mm}$ & - & - & 22. $m /(\mu \mathrm{g})=140 \cdot(D / \mathrm{mm})^{2.70}$ & $r=0.98$ & [Lo] \\
\hline & 17 & $0.50-1.0 \mathrm{~mm}$ & - & - & 23. $v /\left(\mathrm{m} \mathrm{s}^{-1}\right)=0.79 \cdot(\mathrm{m} / \mu \mathrm{gg})^{0.12}$ & $r=0.52$ & [Lo] \\
\hline Lump graupel (R4b) & 116 & $0.40-9.0 \mathrm{~mm}$ & $14-68,000 \mu \mathrm{g}$ & - & 24. $m /(\mu \mathrm{g})=85.0 \cdot(D / \mathrm{mm})^{3.10}$ & 0.89 & {$[H]$} \\
\hline
\end{tabular}

Mitchell (1996) presented fall speed relationships derived from power laws of cross-sectional area and mass vs maximum dimension using a relationship between Re and $X$. We calculate particle mass data from our measurements of maximum 
(5) Plates

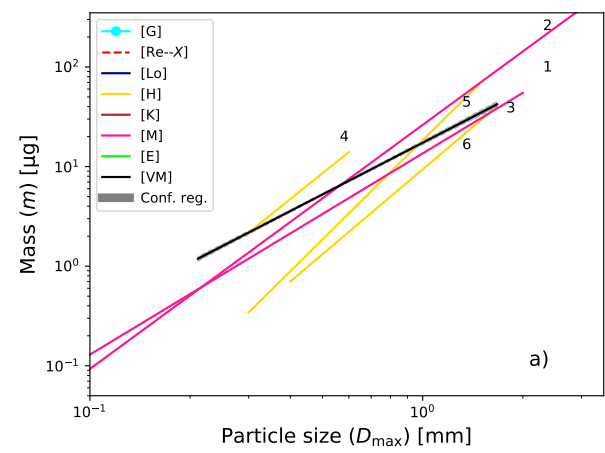

(6) Stellar

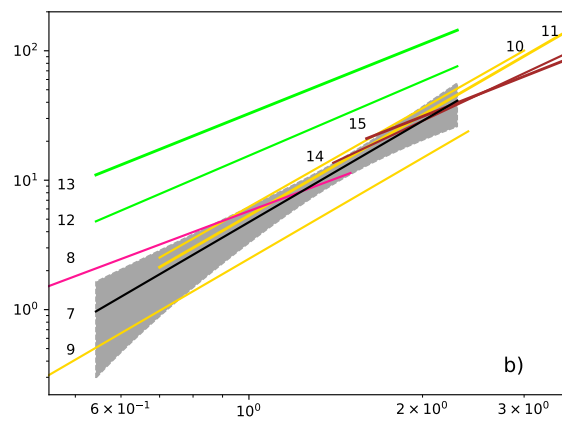

(12) Graupel \& (15) Spherical

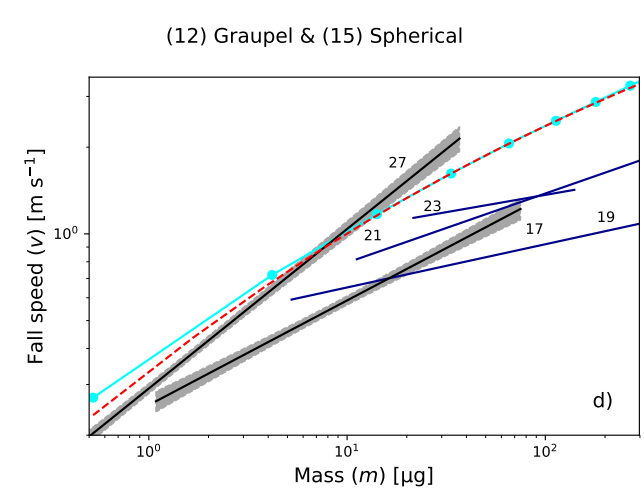

(12) Graupel \& (15) Spherical

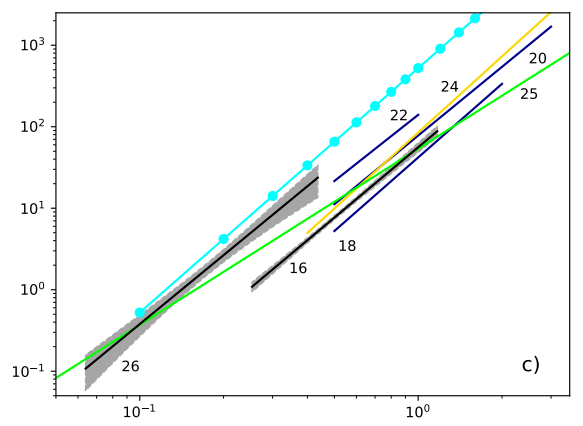

Figure 4. A comparison of the mass vs particle size ( $m$ vs $D)$ and fall speed vs mass $(v$ vs $m$ ) relationships of this study and previous studies for some shape groups: (5) Plates, (6) Stellar, (12) Graupel, and (15) Spherical are shown in logarithmic scale. a)-c) The $m$ vs $D$ relationships for (5) Plates, (6) Stellar, (12) Graupel and (15) Spherical, respectively. d) The $v$ vs $m$ relationships for (12) Graupel and (15) Spherical. For the comparison, parameterizations from Gunn and Kinzer (1949) [G], Locatelli and Hobbs (1974) [Lo], Heymsfield and Kajikawa (1987) [H], Kajikawa (1989) [K], Mitchell (1996) [M], and this work [VM] are shown. In c), the line by [G] corresponds to the mass of spheres given by $m=\frac{\pi}{6} \cdot \rho_{\mathrm{w}} \cdot D_{\max }^{3}$ that was shown also in Fig. 1a). In d), for comparison, a line for speeds determined from Eq. 2 using Re from Eq. 3 and Eq. 4 for spherical particles with density $\rho_{\mathrm{w}}=1 \mathrm{~g} \mathrm{~cm}^{-3}$ is added as a red dashed line. This line is referred to as [Re-X]. These $m$ vs $D, v$ vs $m$ relationships are the same shown and enumerated in Table 2 . The power laws that correspond to [VM] are shown together with their respective $68 \%$ confidence regions. The length of all relationships correspond to the ranges of $D_{\text {max }}$ and $m$ in the $x$-axis (see Table 2).

dimension, cross-sectional area, and fall speed using the same $\operatorname{Re}-X$ relationship. With this new extended dataset, mass vs maximum dimension relationships, mass vs cross-sectional area, and fall speed vs mass, given by Eq. 7-Eq. 9, have been derived and studied for different particle shapes. We present the conclusions that our results led to in this study below.

- As seen in Figs. A1-A3 in Appendix A, and discussed in Section 4.1, the data's large spread is apparent. However, when fitting $m$ vs $D_{\max }, m$ vs $A$, and $v$ vs $m$ relationships to binned data, there are high correlation coefficients for most shape groups, with values between 0.6 and 1 . The only exceptions are shape groups (1) Needles, (2) Crossed needles for the $m$ 
vs $D_{\max }$ relationship with $R_{D}^{2} \simeq 0.2$, and shape group (10) Spatial plates for the $m$ vs $A$ relationship with $R_{A}^{2}=0.45$. For the shape groups with higher values of $R_{D}^{2}$ and $R_{A}^{2}$, i.e. for all groups but (1), (2), and (10), the values of $R_{D}^{2}$ are similar to the corresponding values of $R_{A}^{2}$ (see Fig. 2). For $v$ vs $m$, there is a good correlation for all 15 shape groups (see Table 1).

- When deriving the $m$ vs $D_{\max }, m$ vs $A$, and $v$ vs $m$ relationships analytically from $A$ vs $D_{\max }$ (see Section 3.3), the results are equivalent to fitting to measured data. The analytical relationships Eq. 13-Eq. 15 can be used if power laws are available instead of data. However, fitting to data has the advantage that Eq. 5 can be used rather than power-law approximations required for the analytical derivation of relationships (see B in Appendix).

- The parameters $\tilde{b}_{D}$ and $\tilde{b}_{A}$ are the slopes in the corresponding power laws. Their values are highest for the shape groups (6) Stellar, (11) Spatial stellar, (12) Graupel, and (15) Spherical, close to the values for spheres, i.e. $\tilde{b}_{D}=3$ and $\tilde{b}_{A}=3 / 2$. While this is as expected for shape groups (12) and (15), for groups (6) and (11) it indicates that the morphology in these shape groups remains similar independent of size, i.e. during growth the ice particles grow equally in all three dimensions.

- The exponent values $b_{m}$, i.e. the slopes of $v$ vs $m$, range from 0.33 to 0.55 . These $b_{m}$-slopes do not distinguish the different shapes as seen by the $\tilde{b}_{D}$-slopes for $m$ vs $D_{\max }$. Instead, different speeds at any given mass are characteristic for the different shapes, with the highest fall speed for (15) Spherical and the lowest for (6) Stellar that has shapes with open structures.

- We compared our $m$ vs $D_{\max }$ and $v$ vs $m$ relationships with other mass relationships given by previous studies. The shape groups compared in this study are (5) Plates, (6) Stellar, (12) Graupel, and (15) Spherical. Our results agree reasonably well with the references used.

- For graupel and spheres, (Section 4.4.3), Locatelli and Hobbs (1974) [Lo] reported $m$ vs $D$ relationships for lump graupel (R4b) with different particle densities (high, medium and low). Our relationship for graupel is between [Lo]'s low and medium density relationships, and it is well approximated, by the mass of spherical particles with a density of $0.12 \mathrm{~g} \mathrm{~cm}^{-3}$.

- Looking at $v$ vs $m$, the two lines for spherical particles of $[G]$ and $[\operatorname{Re}-X]$, corresponding to a line for speeds determined from Eq. 2 using Re from Eq. 3 and Eq. 4 for spherical particles with density $\rho_{\mathrm{w}}=1 \mathrm{~g} \mathrm{~cm}^{-3}$, are very close to each other. We report somewhat lower speeds for the shape group (15) Spherical [VM]. This difference may be due to shape group (15) in $[V M]$ consisting of any spherical or almost spherical particle, including ice, whereas the two lines of $[G]$ and $[\operatorname{Re}-X]$ are exclusively for liquid droplets.

These resulting parameterizations may improve our understanding of precipitation in cold climates and improve the microphysical parameterizations in the climate and forecast models. Through these relationships, we can determine particle masses based on fall speed and particle sizes. 
https://doi.org/10.5194/acp-2021-203

Preprint. Discussion started: 16 March 2021

(c) Author(s) 2021. CC BY 4.0 License.

(c) (i)

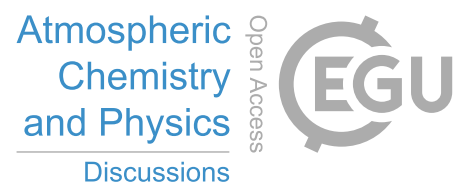

Data availability. The presented data will be available at the Swedish National Data Service (DOI will be available).

340 Author contributions. Conceptualization, T.K., S.V.-M.; resources-instrumentation, T.K.; investigation, S.V.-M., T.K., S.E.; formal analysis, S.V.-M., T.K.; data curation, S.V.-M., T.K.; writing-original draft preparation, S.V.-M.; writing-review and editing, S.V.-M., T.K., S.E.; visualization, S.V.-M.; supervision, T.K., S.E.

Competing interests. The authors declare no conflict of interest.

Acknowledgements. We like to thank the Graduate School of Space Technology at the Luleå University of Technology for financial support 345 and the Swedish Institute of Space Physics (IRF) at Kiruna for offering its facilities for our instrument. 


\section{Appendix A: Mass relationships for the shape groups}

Figures A1-A3 shows the $m$ vs $D_{\max }, m$ vs $A$, and $v$ vs $m$ relationships for all the 15 shape groups fitted to binned data. These relationships correspond to power laws given by Eq. 7-Eq. 9.

\section{Appendix B: Mass derivation using power laws}

The particle mass relationships are derived analytically from a relationship between the Reynolds and Best numbers, in addition to $A$ vs $D_{\max }, v$ vs $D_{\max }$, and $v$ vs $A$ power laws given by Eq. 10-Eq. 12. Section 3 has briefly presented this approach of deriving the particle mass analytically. The $m$ vs $D_{\max }, m$ vs $A$, and $v$ vs $m$ relationships given by this approach are equivalent to fitting to individual data. Indeed we get identical results in the $\tilde{a}_{D}, \tilde{b}_{D}, \tilde{a}_{A}, \tilde{b}_{D}, a_{m}, b_{m}$ parameters if using $X$ vs Re as power law

where $\gamma$ and $\delta$ are the parameters in the power law. We determine these parameters by fitting Eq. 5 to the power law over ranges of Re corresponding to each shape group. For this, we first calculate Re for all particles in a shape group and determine $X$ using Eq. 5 for this set of Re values. Then, we do a linear fit to the logarithm of $X$ vs logarithm of Re. Consequently, for each shape group, we get one set of $\gamma$ and $\delta$.

We express Re as a power law in $D_{\max }$ using Eq. 2 and replacing $v$ with the power law given by Eq. 11

$$
\begin{aligned}
\operatorname{Re}\left(v, D_{\max }\right)=\frac{v\left(D_{\max }\right) \cdot D_{\max } \cdot \rho_{\mathrm{a}}}{\eta}= & \frac{\left(a_{D} \cdot\left(\frac{D_{\max }}{1 \mathrm{~mm}}\right)^{b_{D}}\right) \cdot \frac{D_{\max }}{1 \mathrm{~mm}} \cdot 1 \mathrm{~mm} \cdot \rho_{\mathrm{a}}}{\eta}= \\
& =\left(\frac{a_{D} \cdot \rho_{\mathrm{a}} \cdot 1 \mathrm{~mm}}{\eta}\right) \cdot\left(\frac{D_{\max }}{1 \mathrm{~mm}}\right)^{b_{D}+1} .
\end{aligned}
$$

Now we can determine the particle mass $m$ using Eq. 6 and express it as a function of particle size $D_{\max }$, area $A$, or fall speed $v$. Consequently, the mass relationship as a function of particle size $D_{\max }$ given by Eq. 7 can be derived as follows (using Eq. B1, Eq. B2, and Eq. 10)

$$
\begin{array}{r}
m\left(D_{\max }\right)=\frac{X \cdot A\left(D_{\max }\right) \cdot \eta^{2}}{2 \cdot g \cdot \rho_{\mathrm{a}} \cdot D_{\max }^{2}}=\frac{\eta^{2} \cdot \gamma \cdot\left(\frac{a_{D} \cdot \rho_{\mathrm{a}} \cdot 1 \mathrm{~mm}}{\eta}\right)^{\delta} \cdot a}{2 \cdot g \cdot \rho_{\mathrm{a}} \cdot 1 \mathrm{~mm}^{2}} \cdot\left(\frac{D_{\max }}{1 \mathrm{~mm}}\right)^{\left(b_{D}+1\right) \cdot \delta+b-2}= \\
365=\frac{\eta^{2} \cdot \gamma \cdot a}{2 \cdot g \cdot \rho_{\mathrm{a}} \cdot 1 \mathrm{~mm}^{2}} \cdot\left(\frac{a_{D} \cdot \rho_{\mathrm{a}} \cdot 1 \mathrm{~mm}}{\eta}\right)^{\delta} \cdot\left(\frac{D_{\max }}{1 \mathrm{~mm}}\right)^{b_{D} \cdot \delta+\delta+b-2}=\tilde{a}_{D} \cdot\left(\frac{D_{\max }}{1 \mathrm{~mm}}\right)^{\tilde{b}_{D}} .
\end{array}
$$


The mass relationship as a function of $A$ given by Eq. 8 can be derived as follows (using Eq. B1, Eq. 10, and Eq. 12, and expressing Re as a power law in $A$ )

$$
\begin{array}{r}
m(A)=\frac{X \cdot A \cdot \eta^{2}}{2 \cdot g \cdot \rho_{\mathrm{a}} \cdot D_{\max }(A)^{2}}=\frac{\gamma \cdot \operatorname{Re}^{\delta} \cdot \frac{A}{1 \mathrm{~mm}^{2}} \cdot 1 \mathrm{~mm}^{2} \cdot \eta^{2}}{2 \cdot g \cdot \rho_{\mathrm{a}} \cdot\left[a^{\prime} \cdot\left(\frac{A}{1 \mathrm{~mm}^{2}}\right)^{b^{\prime}}\right]^{2}}= \\
=\frac{\gamma \cdot\left(\frac{v(A) \cdot D_{\max }(A) \cdot \rho_{\mathrm{a}}}{\eta}\right)^{\delta} \cdot \frac{A}{\mathrm{~mm}^{2}} \cdot 1 \mathrm{~mm}^{2} \cdot \eta^{2}}{2 \cdot \rho_{\mathrm{a}} \cdot\left[a^{\prime} \cdot\left(\frac{A}{1 \mathrm{~mm}^{2}}\right)^{b^{\prime}}\right]^{2}}= \\
=\frac{\gamma \cdot\left(\frac{a_{A} \cdot\left(\frac{A}{1 \mathrm{~mm}^{2}}\right)^{b_{A}} \cdot a^{\prime} \cdot\left(\frac{A}{1 \mathrm{~mm}^{2}}\right)^{b^{\prime}} \cdot \rho_{\mathrm{a}}}{\eta}\right)^{\delta} \cdot \frac{A}{\mathrm{~mm}^{2}} \cdot 1 \mathrm{~mm}^{2} \cdot \eta^{2}}{2 \cdot \rho_{\mathrm{a}} \cdot\left[a^{\prime} \cdot\left(\frac{A}{1 \mathrm{~mm}^{2}}\right)^{b^{\prime}}\right]^{2}}= \\
=\frac{\gamma \cdot 1 \mathrm{~mm}^{2} \cdot \eta^{2}}{2 \cdot g \cdot a^{2} \cdot \rho_{\mathrm{a}}} \cdot\left(\frac{a_{A} \cdot a^{\prime} \cdot \rho_{\mathrm{a}}}{\eta}\right)^{\delta} \cdot\left(\frac{A}{1 \mathrm{~mm}^{2}}\right)^{b_{A} \cdot \delta+b^{\prime} \cdot \delta+1-2 \cdot b^{\prime}}=\tilde{a}_{A} \cdot\left(\frac{A}{1 \mathrm{~mm}^{2}}\right)^{\tilde{b}_{A}}
\end{array}
$$

The mass relationship as a function of $v$ given by Eq. 9 can be derived as follows (using Eq. B1, Eq. 11, and Eq. 12, and expressing Re as a power law in $v$ )

$$
\begin{array}{r}
m(v)=\frac{X \cdot A(v) \cdot \eta^{2}}{2 \cdot g \cdot \rho_{\mathrm{a}} \cdot D_{\max }(v)^{2}}=\frac{\gamma \cdot \mathrm{Re}^{\delta} \cdot a_{A}^{\prime} \cdot\left(\frac{v}{1 \mathrm{~m} \mathrm{~s}^{-1}}\right)^{b_{A}^{\prime}} \cdot \eta^{2}}{2 \cdot g \cdot \rho_{\mathrm{a}} \cdot\left[a_{D}^{\prime}\left(\frac{v}{1 \mathrm{~ms}^{-1}}\right)^{b_{D}^{\prime}}\right]^{2}}= \\
=\frac{\gamma \cdot\left(\frac{v}{1 \mathrm{~m} \mathrm{~s}^{-1}} \cdot 1 \mathrm{~m} \mathrm{~s}^{-1} \cdot D_{\max }(v) \cdot \frac{\rho_{\mathrm{a}}}{\eta}\right)^{\delta} \cdot a_{A}^{\prime} \cdot\left(\frac{v}{1 \mathrm{~m} \mathrm{~s}^{-1}}\right)^{b_{A}^{\prime}} \cdot \eta^{2}}{2 \cdot g \cdot \rho_{\mathrm{a}} \cdot\left[a_{D}^{\prime} \cdot\left(\frac{v}{1 \mathrm{~m} \mathrm{~s}^{-1}}\right)^{b_{D}^{\prime}}\right]^{2}}= \\
=\frac{\gamma \cdot\left[\frac{v}{1 \mathrm{~m} \mathrm{~s}^{-1}} \cdot 1 \mathrm{~m} \mathrm{~s}^{-1} \cdot a_{D}^{\prime} \cdot\left(\frac{v}{1 \mathrm{~m} \mathrm{~s}^{-1}}\right)^{b_{D}^{\prime}} \cdot \frac{\rho_{\mathrm{a}}}{\eta}\right]^{\delta} \cdot a_{A}^{\prime} \cdot\left(\frac{v}{1 \mathrm{~m} \mathrm{~s}^{-1}}\right)^{b_{A}^{\prime}} \cdot \eta^{2}}{2 \cdot g \cdot \rho_{\mathrm{a}} \cdot\left[a_{D}^{\prime} \cdot\left(\frac{v}{1 \mathrm{~m} \mathrm{~s}^{-1}}\right)^{b_{D}^{\prime}}\right]^{2}}= \\
=\frac{\gamma \cdot a_{A}^{\prime} \cdot \eta^{2}}{2 \cdot g \cdot a_{D}^{\prime 2} \cdot \rho_{\mathrm{a}}} \cdot\left(\frac{a_{D}^{\prime} \cdot \rho_{\mathrm{a}} \cdot 1 \mathrm{~m} \mathrm{~s}^{-1}}{\eta}\right)^{\delta} \cdot\left(\frac{v}{1 \mathrm{~m} \mathrm{~s}^{-1}}\right)^{\delta+b_{D}^{\prime} \cdot \delta+b_{A}^{\prime}-2 \cdot b_{D}^{\prime}} .
\end{array}
$$

From Eq. B5, we can determine $v(m)$ as follows

$$
\begin{aligned}
& \left(\frac{v}{1 \mathrm{~m} \mathrm{~s}^{-1}}\right)^{\delta+b_{D}^{\prime} \cdot \delta+b_{A}^{\prime}-2 \cdot b_{D}^{\prime}}=\frac{m}{1 \mu \mathrm{g}} \cdot 1 \mu \mathrm{g} \cdot \frac{2 \cdot g \cdot a_{D}^{\prime 2} \cdot \rho_{\mathrm{a}}}{\gamma \cdot a_{A}^{\prime} \cdot \eta^{2}} \cdot\left(\frac{\eta}{a_{D}^{\prime} \cdot \rho_{\mathrm{a}} \cdot 1 \mathrm{~m} \mathrm{~s}^{-1}}\right)^{\delta} \\
& \Rightarrow v(m)=1 \mathrm{~m} \mathrm{~s}^{-1} \cdot\left[\frac{1 \mu \mathrm{g} \cdot 2 \cdot g \cdot a_{D}^{\prime 2} \cdot \rho_{\mathrm{a}}}{\gamma \cdot a_{A}^{\prime} \cdot \eta^{2}} \cdot\left(\frac{\eta}{a_{D}^{\prime} \cdot \rho_{\mathrm{a}} \cdot 1 \mathrm{~m} \mathrm{~s}^{-1}}\right)^{\delta}\right]^{\frac{1}{\left(b_{D}^{\prime}+1\right) \cdot \delta+b_{A}^{\prime}-2 \cdot b_{D}^{\prime}}} \cdot\left(\frac{m}{1 \mu \mathrm{g}}\right)^{\frac{1}{\left(b_{D}^{\prime}+1\right) \cdot \delta+b_{A}^{\prime}-2 \cdot b_{D}^{\prime}}} \\
& =a_{m} \cdot\left(\frac{m}{1 \mu \mathrm{g}}\right)^{b_{m}} .
\end{aligned}
$$



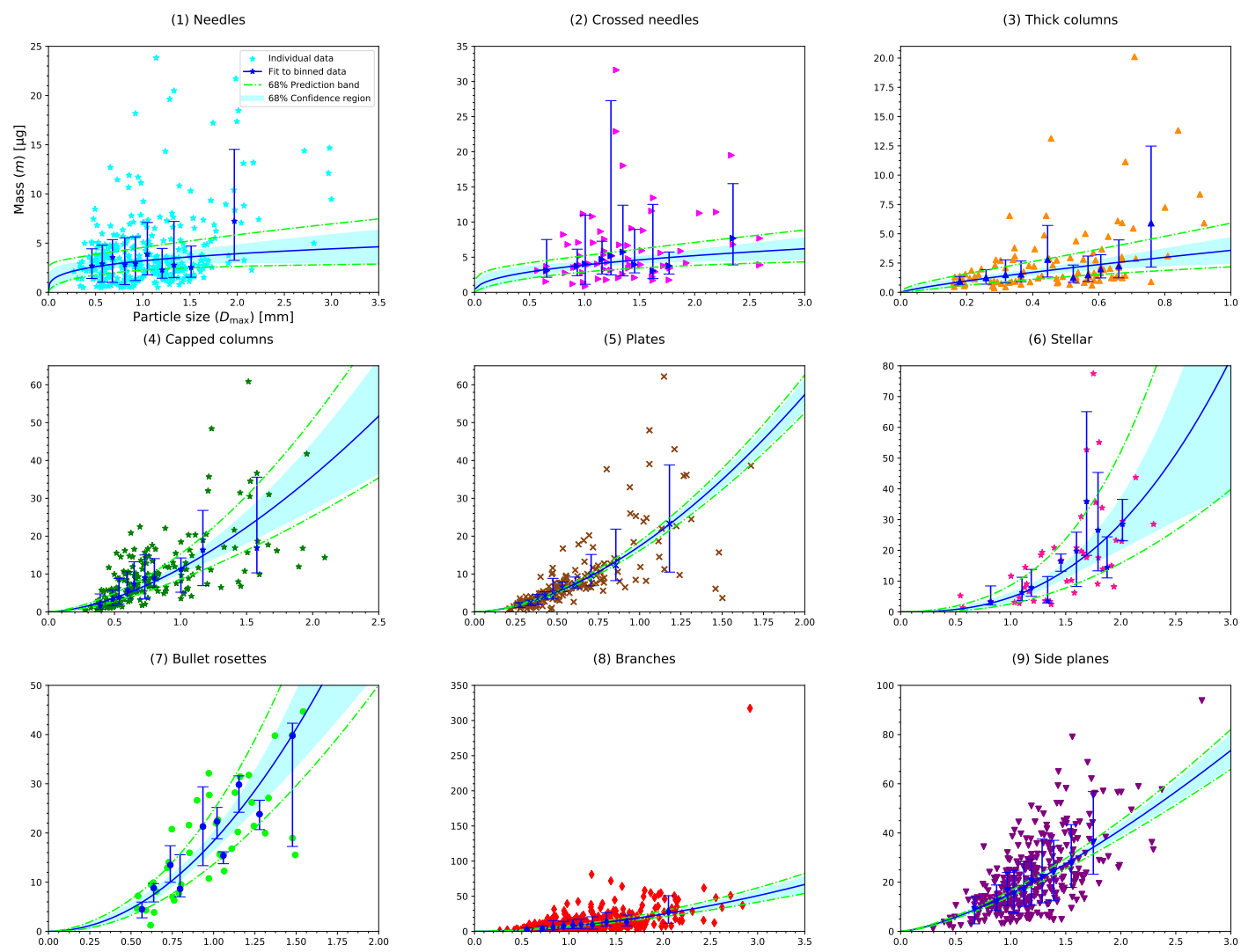

(10) Spatial plates

(11) Spatial stellar
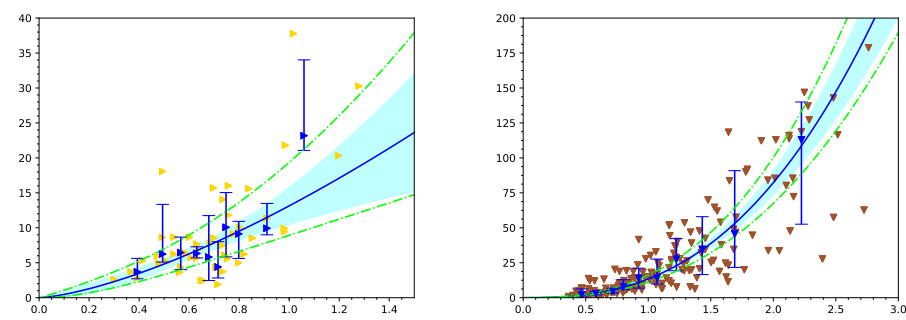

(12) Graupel

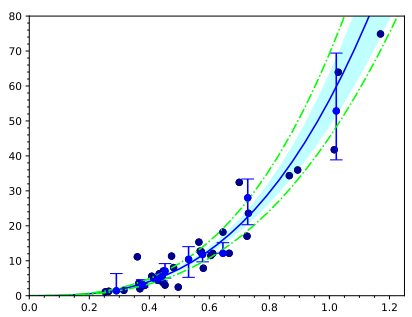

(13) Ice particles
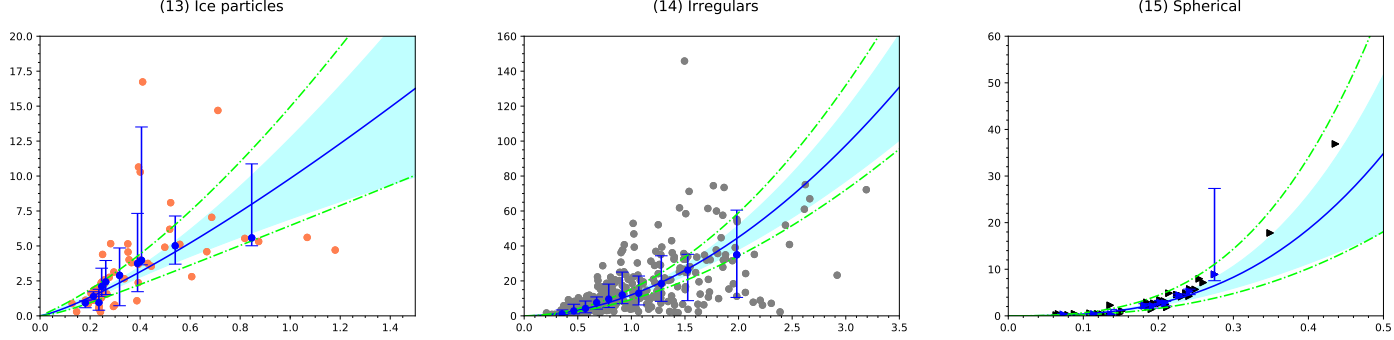

Figure A1. Mass vs particle size ( $m$ vs $D_{\max }$ ) relationships given by Eq. 7-Eq. 9 for all the shape groups are shown. Individual data (coloured symbols) and binned data (blue symbols with error bars) are displayed. Median values in the respective bins represent the binned data. The total length of the error bars represents the spread in mass data, which is given by the difference between the $16^{\text {th }}$ and $84^{\text {th }}$ percentiles. The relationships fitted to binned data are shown. The $68 \%$ prediction band and the $68 \%$ confidence region for the fits are also shown. The same data are shown in Table 1. 
https://doi.org/10.5194/acp-2021-203

Preprint. Discussion started: 16 March 2021

(c) Author(s) 2021. CC BY 4.0 License.

(c) (1)
Atmospheric

Chemistry

and Physics

Discussions
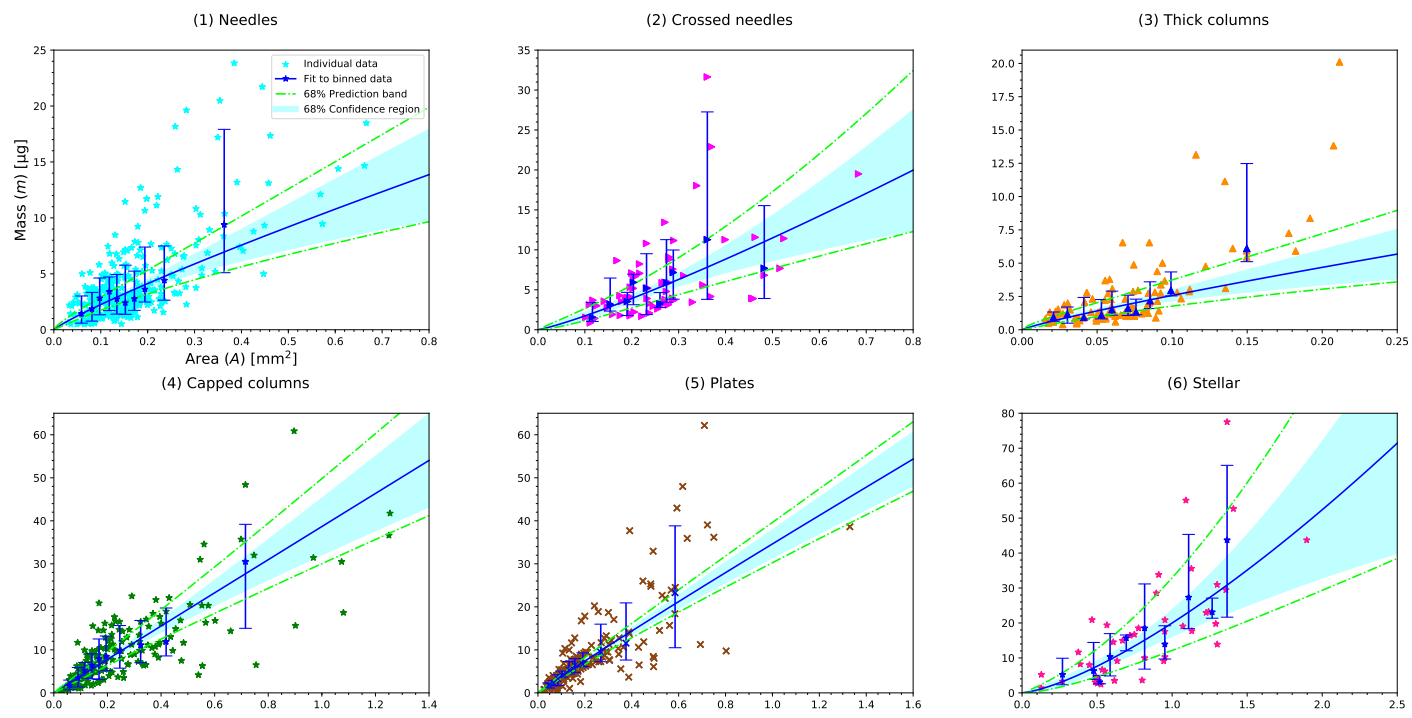

(5) Plates
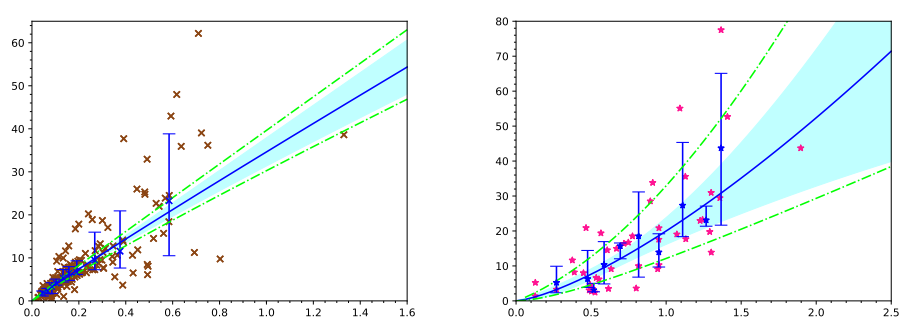

(7) Bullet rosettes

(8) Branches
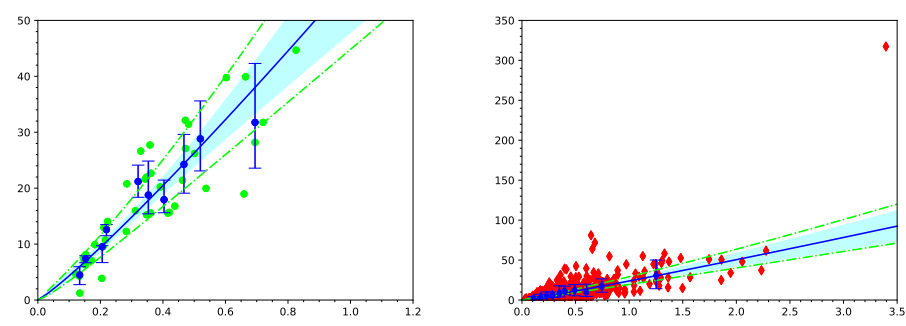

(9) Side planes

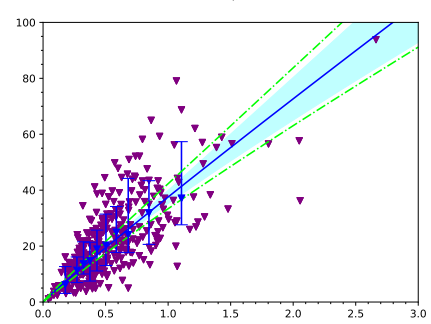

(11) Spatial stellar
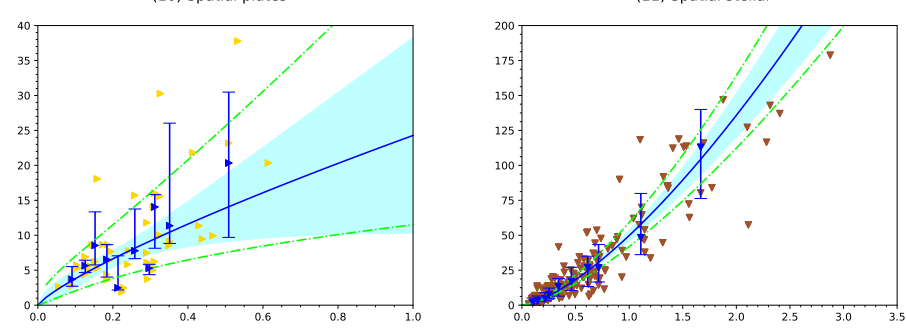

(12) Graupel

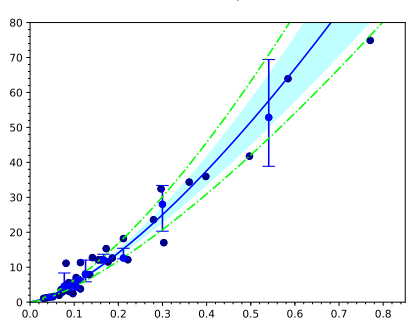

(14) Irregulars
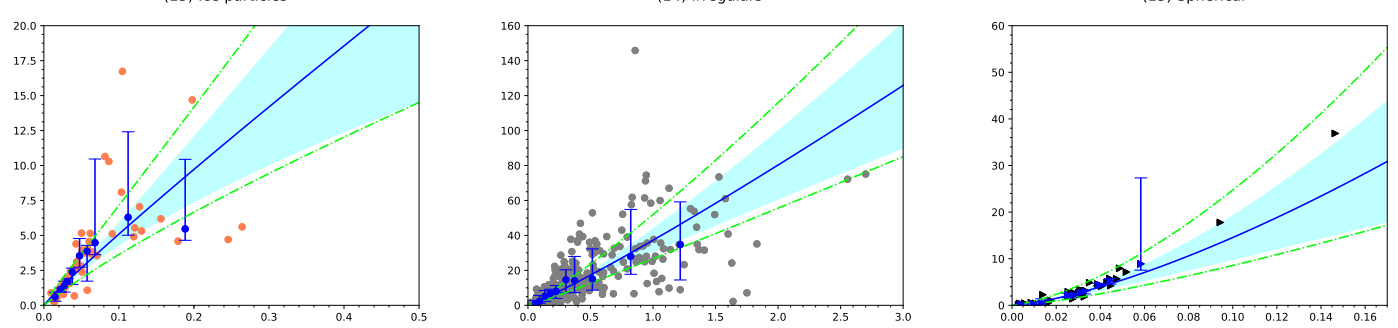

Figure A2. Same as Figure A1, but mass vs cross-sectional area ( $m$ vs $A$ ) relationships given by Eq. 7-Eq. 9 are shown here. 
https://doi.org/10.5194/acp-2021-203

Preprint. Discussion started: 16 March 2021

(c) Author(s) 2021. CC BY 4.0 License.

(c) (1)
Atmospheric

Chemistry

and Physics

Discussions
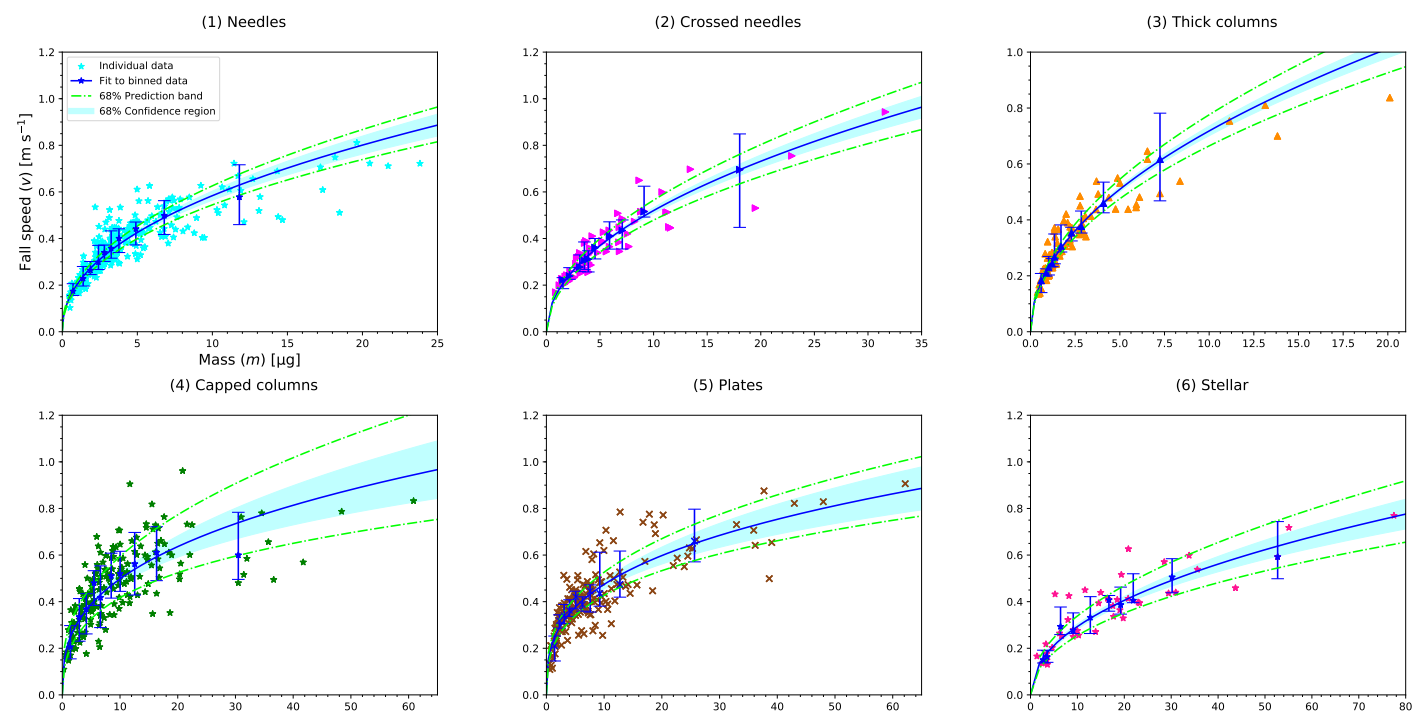

(7) Bullet rosette
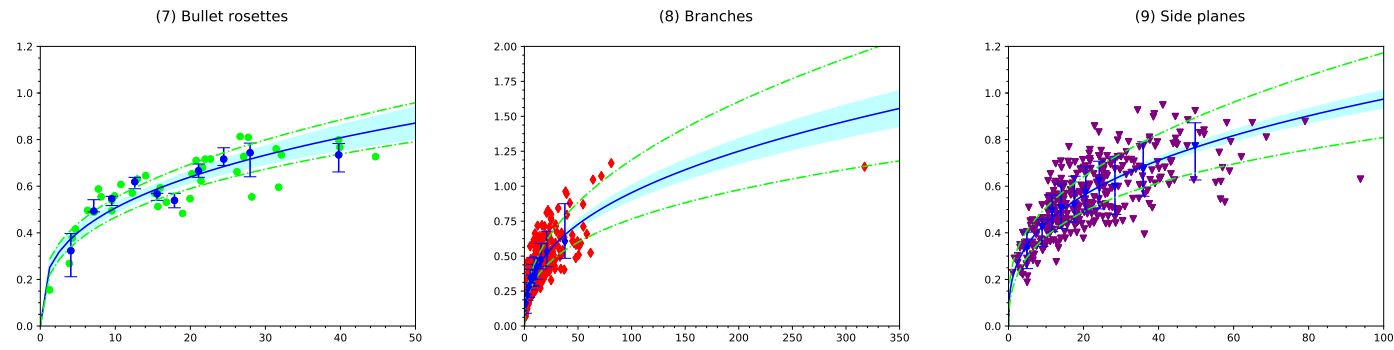

(10) Spatial plates

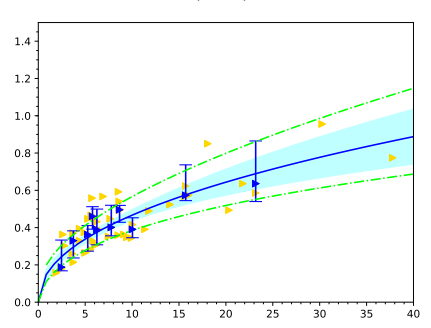

(11) Spatial stellar

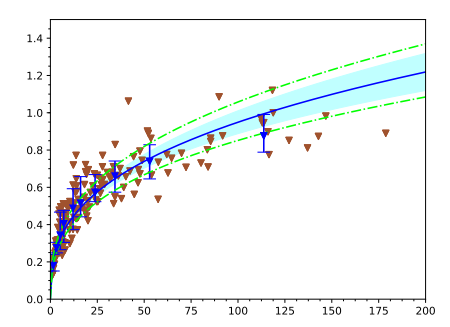

(12) Graupel

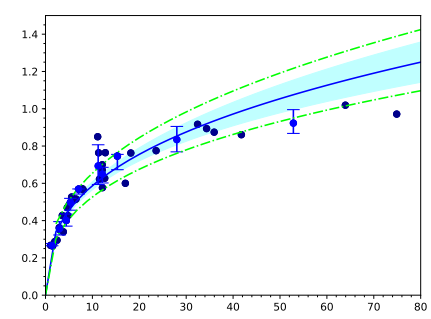

(14) Irregulars
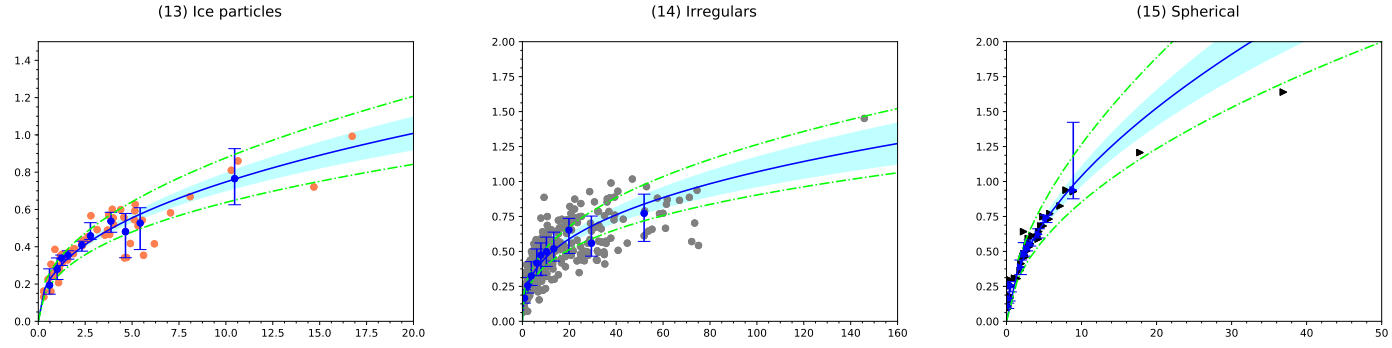

Figure A3. Same as Figures A1-A1, but fall speed vs mass ( $v$ vs $m$ ) relationships given by Eq. 7-Eq. 9 are shown here. 


\section{References}

Magono, C. and Lee, C. W.: Meteorological classification of natural snow crystals, J. Fac. Sci. Hokkaido Univ., Vol. II, 321-335, http: //hdl.handle.net/2115/8672, 1966.

Marchand, R., Mace, G., Hallar, A., McCubbin, I., Matrosov, S., and Shupe, M.: Enhanced Radar Backscattering due to Oriented Ice Particles at $95 \mathrm{GHz}$ during StormVEx, J. Atmos. Oceanic Technol., 30, 2336-2351, https://doi.org/10.1175/JTECH-D-13-00005.1, 2013.

Matrosov, S., Mace, G., Marchand, R., Shupe, M., Hallar, A., and McCubbin, I.: Observations of Ice Crystal Habits with a Scanning Polarimetric W-Band Radar at Slant Linear Depolarization Ratio Mode, J. Atmos. Oceanic Technol., 29, 989-1008,

Abraham, F. F.: Functional Dependence of Drag Coefficient of a Sphere on Reynolds Number, Phys. Fluids, 13, 2194-2195, https://doi.org/10.1063/1.1693218, 1970.

Baum, B. A., Yang, P., Heymsfield, A. J., Schmitt, C. G., Xie, Y., Bansemer, A., Hu, Y.-X., and Zhang, Z.: Improvements in Shortwave Bulk Scattering and Absorption Models for the Remote Sensing of Ice Clouds, J. Appl. Meteorol. Clim., 50, 1037-1056, https://doi.org/10.1175/2010JAMC2608.1, https://doi.org/10.1175/2010JAMC2608.1, 2011. https://doi.org/10.1175/1520-0469(1989)046<2419:AGEFTT>2.0.CO;2, 1989.

Cooper, S., Wood, N., and L'Ecuyer, T.: A variational technique to estimate snowfall rate from coincident radar, snowflake, and fall-speed observations, Atmos. Meas. Tech., 10, 2557-2571, https://doi.org/10.5194/amt-10-2557-2017, 2017.

Erfani, E. and Mitchell, D. L.: Growth of ice particle mass and projected area during riming, Atmospheric Chemistry and Physics, 17,

Gunn, R. and Kinzer, G. D.: The terminal velocity of fall for water droplets in stagnant air, Journal of Meteorology, 6, 243-248, https://doi.org/10.1175/1520-0469(1949)006<0243:TTVOFF>2.0.CO;2, https://doi.org/10.1175/1520-0469(1949)006<0243:TTVOFF> 2.0.CO;2, 1949.

Heymsfield, A. J. and Kajikawa, M.: An Improved Approach to Calculating Terminal Velocities of Plate-like Crystals and Graupel, J. Atmos. Sci., 44, 1088-1099, https://doi.org/10.1175/1520-0469(1987)044<1088:AIATCT>2.0.CO;2, https://doi.org/10.1175/1520-0469(1987) 044<1088:AIATCT>2.0.CO;2, 1987.

Kajikawa, M.: Observation of the Falling Motion of Early Snowflakes., Journal of the Meteorological Society of Japan. Ser. II, 67, 731-738, https://doi.org/10.2151/jmsj1965.67.5_731, 1989.

Kneifel, S., Löhnert, U., Battaglia, A., Crewell, S., and Siebler, D.: Snow scattering signals in ground-based passive microwave radiometer

Kuhn, T. and Vázquez-Martín, S.: Microphysical properties and fall speed measurements of snow ice crystals using the Dual Ice Crystal Imager (D-ICI), Atmospheric Measurement Techniques, 13, 1273-1285, https://doi.org/10.5194/amt-13-1273-2020, https://amt.copernicus. org/articles/13/1273/2020/, 2020.

Locatelli, J. D. and Hobbs, P. V.: Fall speeds and masses of solid precipitation particles, J. Geophys. Res., 79, 2185-2197, Loeb, N. G., Yang, P., Rose, F. G., Hong, G., Sun-Mack, S., Minnis, P., Kato, S., Ham, S.-H., Smith, W. L., Hioki, S., and Tang, G.: Impact of Ice Cloud Microphysics on Satellite Cloud Retrievals and Broadband Flux Radiative Transfer Model Calculations, J. Climate, 31, https://doi.org/10.1175/JTECH-D-11-00131.1, 2012. 
Mitchell, D. L.: Use of Mass- and Area-Dimensional Power Laws for Determining Precipitation Particle Terminal Velocities, J. Atmos. Sci., 53, 1710-1723, https://doi.org/10.1175/1520-0469(1996)053<1710:UOMAAD>2.0.CO;2, https://doi.org/10.1175/1520-0469(1996) 053<1710:UOMAAD>2.0.CO;2, 1996.

Mitchell, D. L., Rasch, P., Ivanova, D., McFarquhar, G., and Nousiainen, T.: Impact of small ice crystal assumptions on ice sedimentation rates in cirrus clouds and GCM simulations, Geophys. Res. Lett., 35, https://doi.org/https://doi.org/10.1029/2008GL033552, 2008.

Schefold, R., Baschek, B., Uest, M., and Barthazy, E.: Fall velocity and axial ratio of snowflakes, Copernicus GmbH, pp. 84-89, 2002.

Stoelinga, M. T., Hobbs, P. V., Mass, C. F., Locatelli, J. D., Colle, B. A., Houze, R. A., Rangno, A. L., Bond, N. A., Smull, B. F., Rasmussen, R. M., Thompson, G., and Colman, B. R.: Improvement of Microphysical Parameterization through Observational Verification Experiment, Bull. Amer. Met. Soc., 84, 1807-1826, https://doi.org/10.1175/bams-84-12-1807, https://doi.org/10.1175\%2Fbams-84-12-1807, 2003.

Sun, W., Hu, Y., Lin, B., Liu, Z., and Videen, G.: The impact of ice cloud particle microphysics on the uncertainty of ice water content retrievals, J. Quant. Spectrosc. Radiat. Transfer, 112, 189-196, https://doi.org/https://doi.org/10.1016/j.jqsrt.2010.04.003, http://www.sciencedirect.com/science/article/pii/S0022407310001391, international Symposium on Atmospheric Light Scattering and Remote Sensing (ISALSaRS’09), 2011.

Szyrmer, W. and Zawadzki, I.: Snow Studies. Part II: Average Relationship between Mass of Snowflakes and Their Terminal Fall Velocity, J. Atmos. Sci., 67, 3319-3335, https://doi.org/10.1175/2010JAS3390.1, https://doi.org/10.1175/2010JAS3390.1, 2010.

Tao, W.-K., Simpson, J., Baker, D., Braun, S., Chou, M.-D., Ferrier, B., Johnson, D., Khain, A., Lang, S., Lynn, B., Shie, C.-L., Starr, D., Sui, C.-H., Wang, Y., and Wetzel, P.: Microphysics, radiation and surface processes in the Goddard Cumulus Ensemble (GCE) model, Meteorol. Atmos. Phys., 82, 97-137, 2003.

Thornberry, T. D., Rollins, A. W., Avery, M. A., Woods, S., Lawson, R. P., Bui, T. V., and Gao, R.-S.: Ice water content-extinction relationships and effective diameter for TTL cirrus derived from in situ measurements during ATTREX 2014, Journal of Geophysical Research: Atmospheres, 122, 4494-4507, https://doi.org/10.1002/2016JD025948, https://agupubs.onlinelibrary.wiley.com/doi/abs/10.1002/ 2016JD025948, 2017.

Vázquez-Martín, S., Kuhn, T., and Eliasson, S.: Shape Dependence of Falling Snow Crystals’ Microphysical Properties Using an Updated Shape Classification, Appl. Sci., 10, 1163, https://doi.org/10.3390/app10031163, 2020a.

Vázquez-Martín, S., Kuhn, T., and Eliasson, S.: Shape dependence on fall speed of snow crystals, Atmos. Chem. Phys. Discuss., pp. 1-32, https://doi.org/10.5194/acp-2020-1056, 2020b.

Waliser, D. E., Li, J.-L. F., Woods, C. P., Austin, R. T., Bacmeister, J., Chern, J., Del Genio, A., Jiang, J. H., Kuang, Z., Meng, H., Minnis, P., Platnick, S., Rossow, W. B., Stephens, G. L., Sun-Mack, S., Tao, W.-K., Tompkins, A. M., Vane, D. G., Walker, C., and Wu, D.: Cloud ice: A climate model challenge with signs and expectations of progress, Journal of Geophysical Research: Atmospheres, 114, https://doi.org/10.1029/2008JD010015, https://agupubs.onlinelibrary.wiley.com/doi/abs/10.1029/2008JD010015, 2009.

Xie, Y., Yang, P., Kattawar, G., Baum, B., and Hu, Y.: Simulation of the optical properties of plate aggregates for application to the remote sensing of cirrus clouds, Appl. Opt., 50, 1065-81, https://doi.org/10.1364/AO.50.001065, 2011.

Yang, P., Zhang, Z., Kattawar, G. W., Warren, S. G., Baum, B. A., Huang, H.-L., Hu, Y. X., Winker, D., and Iaquinta, J.: Effect of Cavities on the Optical Properties of Bullet Rosettes: Implications for Active and Passive Remote Sensing of Ice Cloud Properties, J. Appl. Meteorol. Clim., 47, 2311-2330, https://doi.org/10.1175/2008JAMC1905.1, 2008. 